Volume 18, Number 1, Winter 1989

ISSN $0710-4340$

The Future of Educational Technology is Past

P. David Mitchell

Modifying the Regulation Processes of Learning:

Two Exploratory Training Studies

P. R. J. Simons

Applications and Implications of Distance Education:

Manitoba

Bernard Simand

Televised Instruction for Post-Secondary

Education in Québec

Gilles Carrier

Mark Schofield

The Slope Test: Applications in Formative Evaluation

Jon Baggaley

Aaron-Henry Brauer

An AMTEC Publication 
EDITORIAL BOARD

Jon Baggaley

Concordia University

F. Bamy Brown

University of Saskatchewan

Elaine Bruce

Concordia University

Bruce Clark

University of Calgary

Dan Coldeway

Afhabasca University

George L. Gels

OISE

W. D. Godfrey

Softwords

William Hanson

Calgary Board of Education

William Hillgartner

McGill University

Denls Hlynka

University of Manitoba

Robert Jones

Sheridan College of Applied

Arts and Technology

Richard F. Lewis

University of Windsor

Hanna Mayer

Athabasca University

Tom Rich

Prince Edward Island Department of Education

Alexander J. Romlszowski

Syracuse University

Lauran Sandals

University of Calgary

Richard F. Schmld

Concordia University
Richard A. Schwier

University of Saskatchewan

Marlela Tovar

Concordla University

Jesus Vazquez-Abad

Les Technologies EDINOV

Cynthia B. Weston

McGill University

Laura R. Winer

APO Quebec

AMTEC BOARD OF

DIRECTORS

President

Wayne Blair

Alberta Department of

Education

Past President

June R. Landsburg

Simon Fraser University

President Elect

Mary Kennedy

Memorial University

Secretary/Treasurer

Ron Eyre

The Wellington County Board of Education

\section{Director}

Margaret Sadler

University of Alberta

\section{Director}

Al LeBlanc

Sault Ste. Marie Board of Education

\section{Director}

Dave Bleman

Huron County Board of

Education 


\section{Canadian Joumal of Educational Communication}

Volume 18, Number 1

Winter 1989

Editor

Robert M. Bernard

Assistant Editor

Mandie Aaron

Production Manager

Mary Genova, WRITEWORKS

Editorial Assistant

Jacques LeCavalier

Production Assistant

Michele Gour

ISSN $0710-4340$

The Canadian Joumal of Educational Communication is published quarterly by the Association for Media and Technology in Education In Canada; 500 Victoria Road North; Guelph, Ontario N1E 6K2; Canada; Attention: Mr. Ron Eyre, Secretary/Treasurer. Notification of address change should be sent to the above. All articles are copyright by AMTEC and may be reproduced for nonprofit use without permission provided credit is given to CJEC. Back issues of CJEC are $\$ 10$ Canadian and may be obtained by contacting the Editor. CJEC is indexed in the Canadian Education Index and ERIC.

Second Class Mail Registration No. 6956

\section{PERSPECTIVE}

The Future of Educational

Technology is Past

P. David Mitchell

\section{ARTICLE}

Modifying the Regulation

Processes of Leaming: Two

Exploratory Training Studies

P. R. J. Simons

\section{PROFILES}

Applications and Implications of

Distance Education: Manitoba

Bemard Simand

Televised Instruction for Post-Secondary Education In Quebec

Gilles Camier

Mark Schofield

\section{RESEARCH TECHNIQUE}

The Slope Test: Applications

in Formative Evaluation

Jon Baggaley

Aaron- Henry Brauer

MEDIA MANAGERS COLUMN

Letting AV Media Work for You 69

by Jarvis Stoddart

BOOK REVIEWS

Three ERIC Monographs are

reviewed by Gary Boyd,

Cheryl Amundsen and

Jon Baggaley

All correspondence should be addressed to:

Dr. Robert M. Bemard, Editor

Education Department

Concordia University 1455 de Maisonneuve Blvd. W. Montreal, PQ, Canada H3G 1M8 


\section{CJEC ORDER FORM}

CJEC is free to members of AMTEC. However, many prefer

to order the journal separately, and may do so by completing

the form below:

(Name)

(Address)

\section{Acknowledgement}

The Canadian Journal of Educational

Communication is supported by a grant from the Social Sciences and Humanities Research Council of Canada.

CJEC is typeset on an Apple Macintosh Phus(tm) in PageMaker 3.01(tm). Galley proofs to authors and final cameraready impressions are output on an Apple Laser Plus Writer(tm).

Printing: Concordia University

$\$ 40.00$ (Cdn.) annual subscription

New Subscriber

Renewal

Please send me information about AMTEC and a membership form.

CHANGE OF ADDRESS

\begin{tabular}{|l|}
\hline \\
\hline \\
\hline \\
\hline Sendthlsformto: \\
Mr. Ron Eyre \\
AMTEC \\
500 Victoria Road North \\
Guelph, Ontario N1 E \\
Canada
\end{tabular}


Perspective

\title{
The Future of Educational Technology is Past
}

\author{
P. David Mitchell
}

\begin{abstract}
Editor's Note: This is the third in a series of invited artic les that are published in CJ EC in Number 1 of each new volume. These articles are intended to serve as a mechanism for addressing the broader issues In educational communication and technology and for challenging our assumptions about the undenlying nature and current state of our profession and the professional activities in which we engage. In this year's Perspective P. David Mitchell argues that the traps referred to by Beckwith (Perspective. 1988) are unavoidable and that in effect the promise of educational technology as envisioned by Beckwith and others is dead - killed largely by our inability or unwillingness to examine the underlying tenets of our own behavior and to affect change in the processes and practices that have become the field of educational technology. We must adopt a new perspective on the process of leaming, the process of teaching, and the process of doing research, he argues, if we are to revive the corpse before It is buried by someone else,
\end{abstract}

\section{PROLOGUE}

Educational technology appears to be a successful field. Graduates are in high demand, working primarily in industrial training and the formal education system. Salaries and opportunities for advancement apparently are good. New and promising equipment appears on the market regularly, awaiting our exploitation. Educational technology journals and conferences abound. People in traditional disciplines and professions are using some of our "tricks of the trade" and fellow academics recognize the value of educational technology- in short, educational technology seems to be in its prime and enjoying good health. What, then is the meaning of the title?

This paper is an attempt to share my concern about the value of the field of educational technology to our society, especially to those currently in school and university, and to the global society within which we function. It also is an attempt to expand upon the cogent analysis and hopeful prescription of Beckwith (1988), an expansion which will show the impossibility of escaping the traps which he describes. I write as an insider, one who has devoted two decades to helping fulfill Kenneth Richmond's prediction that "educational 
technology is destined to emerge as the central humane discipline of the future" (Richmond, 1967, p. 106). And I write as one who has worked within the philosophical and systemic perspective that Beckwith insists we adopt, as well as within a graduate programme preparing educational technologists.'

Despite my frequent attempts to maintain a balanced perspective on issues, I'm not always antifloccinaucinihilipilificationistically inclined. (Note to proofreader: see OED if spelling checker program chokes on this!) So it is with regret that I now conclude that educational technology has no future because it is dead (though not yet buried). Any hope for its resuscitation is likely to be misplaced because there is so little understanding of why it died. I hope that this post mortem analysis will reduce our lack ofunderstanding and perhaps contribute to a new life.

In preparing this paper I attempted to raise many questions and to suggest few answers. Moreover, I am aware that most complex problems have many solutions-or none -and that suggested answers are not final. Some comments are deliberately provocative and are intended to stimulate critical discussion; others appear so in the absence of elaboration.

To illustrate, I intend to show that we have failed to tackle the most pressing educational problems and have settled for routine applications more characteristic of a craft. Moreover we have developed virtually no theoretical models (those we use tend to be borrowed) nor do we produce graduates who are likely to do so. The underlying reasons are complex but centre on our adopting a world view that is, if not obsolete, incomplete and useless for understanding the complex problems that need to be solved. Many have argued that we need a new paradigm but this calls for each of us to transform ourselves. We lack the requisite psychotechnology to make this easy. Paradoxically we need this paradigm in order to acquire it.

\section{THE FUTURE OF EDUCATIONAL TECHNOLOGY}

In his paper portraying "The Future of Educational Technology," Beckwith argues that, "If we are to survive, purposefully, as the cutting-edge field of our original vision, there are some solution-related traps that must be avoided" (Beckwith, 1988, p. 8). These he classifies as the traps of: "compromised integrity"; "status quo adherence" and "solidification."

In the first trap, we are distracted from actually achieving our educational goals by, for example, dazzling products, pursuit of money, or our employer's goals. In the second, we seek credibility by emulating established professions' inertia. Thus we avoid recommending significant changes in any educational system which employs us to solve a problem. And our notion of acceptable research or conferences is governed by the norms established by others (e.g., psychology).

In the trap of solidification, "The energy needed to apply the intellectual techniques of educational technology to the betterment ofhumankindhas been 
sapped by solidification within the mode of short-term survival" (Beckwith, 1988, p. 13). The purpose of educational technology becomes lost within the mists of routine applications of standard (though not necessarily valuable) procedures. Thus graduate programmes focus on instructional design and research comes "close to solidification as an inappropriate and limited method of inquiry. The cementing of reductionist, conclusion-oriented, static, systematic research models precludes the needed study and realization of systemic entities" (p. 14). Is there any hope? Beckwith thinks so.

His solution is deceptively simple. We need to publicly proclaim our goal to be "the transformation of learners and learning processes" and we need to transform our field into a systemic field which itself could become "the personal learning environment of your dreams - rich, vibrant, alive, dynamic, accelerating- an environment in which . . . research and development, production and dissemination, and teaching and learning are fused so tightly together that transformation is activated and reactivated (Beckwith, 1988, p. 17). Having suggested similar ideas myself (Mitchell, 1970; 1971; 1975; 1978; 1982) I must admit that this vision is appealing. But is it realistic?

\section{ACTIONS SPEAK LOUDER THAN WORDS}

These are not traps to be avoided; they are symptoms ofincurable terminal illness. Moreover, the problem is not confined to educational technologists. For most organizations that employ educational technologists, education is no longer the system's purpose; what happens to students is just a by-product of the activity of its professional and bureaucratic core. This is a startling comment to which I'll return later.

Have you ever stopped to consider that perhaps what some of us are doing ought not to be done at all? And other things might be accomplished better by technicians, paraprofessionals and sundry other assistants. How are we to prepare ourselves for future developments (e.g., in micro-electronics, political struggles for declining budgets, cybernetics) when we don't even know what to anticipate? Are some of us failing to do what ought to be attempted and, if so, how do we identify the requisite capability in order to transform the field and to prepare new practitioners of educational technology to undertake these important tasks?

Earlier I asserted that in most organizations that employ educational technologists education is no longer the system's purpose ; what happens to students is just a by-product of the activity of its professional and bureaucratic core. What do I mean? Simply put, a system's purpose can be better discerned by asking what the system is doing, not what it was intended to do or what its spokesmen claim it's doing.

Typically, the system's core is devoted to self-perpetuation of their roles and functions (no matter how well-meaning the people are). What they do defines the system's purpose (cf. Beer, 1986). Thus, teaching becomes defined 
by philosophers and teachers as what teachers do regardless of whether students learn or even attend school (truancy rates run as high as $30 \%$ in some places). Health care becomes defined as what doctors provide (despite findings that nearly half the medical problems may be produced by doctors). Education is defined as happening in schools and colleges despite the prevalence of near illiteracy and limited knowledge or skills amongst students and even graduates. And what about educational technology? There is a dangerous precedent for defining it as what practitioners do; function becomes purpose.

Is this radical approach to purpose reasonable? How realistic is it to think that education is a high priority in the typical school or university? If a visiting scientist from Mars were to visit your institution and attempt to infer that system's purpose by observing how people spend their time and how money is allocated, would he infer education to be its primary purpose? Or would he take the extreme view that, "Universities are machines created by their gods, the faculty, primarily to provide them with the quality of work life they desire. Education of students is the price they must pay for this privilege. Teaching is largely devoted to inculcating students with a vocabulary that enables them to speak authoritatively on subjects they do not understand" (Gharajedaghi \& Ackoff, 1985, p. 22). These authors go on to conclude that, "Schools in general, and universities, colleges and departments in particular are organized bureaucratically, that is, mechanistically They strongly resist innovation. They restrain their employees with rigid rules and regulations." (Gharajedaghi \& Ackoff, 1985, p. 23).

Let me give you a humorous but true example which illustrates my point that its purpose is what a system does. A Ph.D. student registered at a certain American university filled out an application form and indicated her first languages were Arabic, Armenian, English, French and Turkish. Later, when the second language requirement for the Ph.D. had to be satisfied - an educational objective intended to guarantee that the student could read work written in another tongue - she was told that since all of these were first languages she must take a second language. Undaunted, she pointed out that computer languages could count and she knew both COBOL and FORTRAN. Equally undaunted, bureaucracy said that they did not count because she had studied them as an undergraduate and already had received credit for them. So she had to study and pass an exam in German! What's the point?," you may wonder. Just this: too frequently educational technologists behave unwittingly like this bureaucrat.

We do so when we try to improve the operation of an existing system without considering its actual and intended purpose; (Do your administrators and colleagues really act as if education were the prime purpose school, college or training unit? Does the Ministry of Education? Do your students? Do you? Would your time be better spent doing something else?)

We do so when we try to operationalize important educational intentions by composing and writing behavioural objectives, the sum of which falls short of the envisioned end-stage (e.g., the "good doctor" or the "good educational 
technologist" is a person who is far more than the component objectives of his professional courses).

We do so when we decide that we will produce a film or a series of TV programmes and look around for a topic rather than looking for an educational problem that needs to be solved and undertaking analysis to see which media/ methods/content mix is most propitious.

We do so when we design so-called individualized instruction that fails to take into account the idiosyncratic background and learning styles of students and the network structure of knowledge in the discipline that would allow a student to build better conceptual links between what he knows and what he needs to know.

We do so when we design research projects that contribute little or nothing to the theory or practice of education but simply show our prowess as surveyors or experimenters.

And most pertinent here, we do so when we think a common preparation for educational technologists would look like the course of study we followed. What is the intended purpose of a system that produces educational technologists? What kind of person do we hope to turn out and what will that person need to know, believe, hope, fear, love and do? Expressed otherwise, what can an educational technology program do for society?

\section{THE CHALLENGE TO EDUCATIONAL TECHNOLOGY}

Educational technology must be dedicated to the efficiency of education as a whole and not simply to specific operations. An operational and philosophical analysis of educational technology, calls for a consideration of overall problems of education which educational technologists may be able to tackle before proceeding to the lower-order problem of designing a curriculum for them. The field of educational technology -in its concern for the optimal organization of education - must not be limited to time-honoured structures. Not should it perpetuate failures. We might serve our stewardship better by devising activities and forming environments which permit people to live fully and intensely both within and outside so-called educational institutions.

While rich countries provide tax-supported schooling for 12 to 20 years, half the world's children cannot attend school. This paradox underscores the need for change. The world's education system grinds on, consuming everincreasing amounts of money in response to demand for educational services regardless of whether education is the outcome. But school costs in both affluent and penurious nations rise more rapidly than enrolments or national incomes. No country in the world can afford to satisfy its educational needs by schooling alone. Does educational technology offer any hope?

The Sisyphean educational imperative is to provide access to stored human experience - ideas, knowledge, skills - and opportunities to develop what is needed for personal and cultural development. 
Enormous problems must be solved ifmankind as a whole is to share in the potential for human comfort, achievement and eudaemonia now restricted to a tiny minority. To refurbish our ideas about how to implement man's educational aspirations we need to develop the requisite theory and practice of educational technology along the lines suggested by Beckwith, but going much farther. Then educational technology can achieve the lofty ideals ascribed to by many of us.

The concept of lifelong education provides an altered perspective of profound significance for educational technology. Changing from dedication to efficiency ofinstructional activities to dedication to the effectiveness of human existence - which is what education entails - may reflect less a change in intellectual and communications technology than in priorities. But it calls for a new paradigm too.

Education refers to certain activities concerned with the intentional organization of ideas and learning opportunities by which successive generations are encultured and trained to sustain themselves and contribute to society. Continuing education presents two challenges. How can each nation enhance its collective intellectual capacity and skills? How can each person develop his personality and meet his educational and cultural aspirations?

Two currents of thought, the one emphasizing education as a productive investment for development of society and the other emphasizing personality development need to be combined. What is the scope of education today?

\section{WHAT IS AN EDUCATIONAL PROBLEM?}

If educational technology is to contribute to the solution of educational problems, we must first come to grips with the scope and purpose of education. Educational technology then may be in a position to identify worthwhile solutions.

The essence of technology, and therefore educational technology, is knowledge about relationships. Thus if we perform action $\mathrm{X}$, there is a probability, $\mathrm{P}$, that a given outcome, $\mathrm{Y}$ will occur. Alas in education and training it seldom is clear what actionX is most likely to produce the intended result Y, especially without also producing unintended and conflicting outcomes. Moreover, $\mathrm{Y}$ is seldom unambiguous and confounds different, even incompatible, goals (e.g., attempting to optimize the state of interacting subsystems). Recall that the term education incorporates at least two different concepts; the personal experiences of someone coming to understand or appreciate or reflect upon the world; and the organized attempt to produce those experiences in a number of other persons. How can we optimize both? (Operational research holds some promise for systemic analysis in this area but we have yet to see much in the way of results.) 


\section{The Scope of Education}

An educational problem may be far greater than the restricted vision of many observers. Thus an instructional design problem may be considered in isolation but the instructional system itself is embedded in an organization (school, corporation) that has other subsystems with different goals, priorities and resources that interaet with it.And this organization, in turn, is embedded with other interacting subsystems in a larger system. Ib complicate matters even more, each learner has his/her own system of knowledge, values, goals, etc.

In short, the notion of an educational problem or system should be expanded to include more systems and subsystems. And the boundaries between activities that are labelled educational and those that are not, should be pushed back to encompass informal as well as directed learning. Think for a moment about where you learned most of your attitudes, knowledge and skills. Was it exclusively, or even largely, within institutions labelled educational? Our classical methods of dealing with educational problems cannot be expected to be of much use in tackling such systemic problems.

\section{A Larger Perspective}

The world is in a critical phase of its evolution. Astonishing changes in micro-electronics and information technology presage new structures in many areas. We are promised that robots will produce half of our manufactured goods, displace human labour (including cheap labour in the third world) and send countless adults back to school. The opportunity for untold wealth is nigh; so is the possibility of disaster. Various reports suggest that continuous education soon will be a form of universal occupation. Opportunities for educational technology seem endless. Yet most people in the world live in the stick age; they get their energy from burning sticks and their life style centres on hand hoe agriculture. Their children die from malnutrition and disease (both of which are linked to poverty as well as to inadequate education) or military action. They strive for self-sufficiency constrained by their environment. We, in Canada, confront what some fear could herald a return to that life style (insofar as massive unemployment might reduce our economy to a shambles) and others hope could offer a culturally rich and personally rewarding life style. Surely there are real and challenging problems for us to attack. Perhaps, as Schwen suggests, "Our conceptual process traditions will be the most sustaining or enduring approach to solving problems" (1988, p. 25).

Our leaders in government, industry and education face many complex, inter-lockingproblems and possibilities. We are immersed in an era of unprecedented changes in what is possible and in the physical and psychological environment as a result of our decisions. Perhaps most significant is the increasing rate of change. We have just become accustomed to the silicon chip and now must adapt to a protein chip that promises to increase the density of a chip by 100,000 times.Add to this the possibility of neural net computers. Can we even conceive of the potential impact of such a development on education 
and training? The need for educational technology (in Beckwith's sense) has never been greater. Yet educational technology probably cannot be revived to tackle these complex problems. Computer scientists will be asked to do so.

\section{TOWARD A SYSTEMIC PERSPECTIVE}

\section{The Input-Output Model}

Most definitions of educational technology assert that it is concerned with applying knowledge, systems and techniques to improve the process of human learning. But virtually all educational technology research and applications have attempted instead to improve instruction, especially through information display systems and clarification of objectives as observables. Even interactive systems over-rely on information retrieval and display rather than responding to what the learner understands about the subject. Educational technology has turned the learner into a programmable machine rather than developing support systems to improve the quality of learner/subject matter interactions. This is primarily because we have failed to reject the notion that teaching causes learning and adhere to a simple cause-effect paradigm.

If we consider the various paradigms that have influenced educational technology, we see that they have been analytic and reductionistic even though different on the surface. Whether we consider the audio-visual, behavioural, neo-behavioural or cognitive models, all treat the learner as an input-output system which somehow responds to information displays by means of (potentially) measurable changes in capability. When we notice differences in learners'behaviour we attempt to relate these to factors under our control (e.g., message design, reinforcers) or uncontrolled variables (e.g., internal vs. external locus of control, gender, learning strategy).

For each of these paradigms the over-riding problem is how the educational system ought to work, both in general and specifically for an identifiable group of learners. This, in turn, leads to the notion that some human being (e.g., educational technologists, trainers, teachers) are expected to apply these causative factors (objectives, advance organizers, instructional materials, rewards or punishments) to other human beings. This is not as simple in practice as one might wish.

Experimental research controls the influence of the environment in order to predict events; a complex, adaptive environment confounds such models. Thus if we perform action X, not only is there a probability of outcome Ybut this action, in turn, generates a cascade of events, some of which may alter $\mathrm{X}$-and thereby alter Y- until the loop is broken. The traditional cause-effect model is useful only up to a point. The environment-free concept of explanation fails to provide an understanding of complex systems of the sort that educators deal with. But there is another problem with the cause-effect model. 


\section{Goal-Directed Feedback}

What does it mean to be "in control?" In order for a teacher or instructional system to teach (i.e., to control a student's behaviour) the controller must be able to generate or select a desired outcome (e.g., a set of behavioural objectives), discriminate between what is observed to happen and what is intended to happen, and select actions which reduce the discrepancy This is easier to imagine with a human teacher or computer aided learning than with a book or television program but the principle still applies in a modified form.

The model case is a control system designed by an engineer. He knows that there is a control system and knows what it controls. Moreover, he knows that the controlled system can be controlled because of the way it is designed. In other words, the controller must operate according to principles that do not apply to the system being controlled ( $c f$. Powers, 1973). While this may be appropriate for inanimate systems, how useful is it when considering humans? Are we justified in using two models of human behaviour, one for those who control and one for the persons being controlled?

In most educational technology research and practice this seems to be exactly what happens. Perhaps this trap is a legacy from psychological research where the experimenter is presumed to be controlling the organism's behaviour (despite the dim awareness, albeit in cartoons, that the rat pressing a lever is controlling the food-givingbehaviour of the psychologist). Or perhaps it is a legacy from the days of birch and leather teaching aids. Control theory offers an escape from this trap.

\section{Control System Theory}

Control theory seems to have originated four decades ago though its roots are ancient. Norbert Wiener's (1948) seminal work on cybernetics introduced a new paradigm for understanding human nature, indeed all organisms, whether we view them as agents or objects of control. Cybernetics, he showed, was concerned with control in, not control of, the organism or machine. Because "control" sounds manipulative, even authoritarian, we may wish to substitute "regulation" for it. But control theory has emerged as the theory of systems which control rather than a theory of how to control other systems. The distinction is not as subtle as the wording might suggest. Indeed the fundamental ideas ofcontrol theory have the potential to produce the transformation in our thinking about education, indeed of society, that Beckwith insists is needed. More important it can alter our own perspective on education.

In the first place, if we begin to take into account the controlling behaviour of the people previously thought of only as objects to be controlled, whether in experiments, in the classroom or by a computer tutor, we immediately can see that learners'ability to control themselves is essential to education. Moreover, the fundamental observable is not the simple cause-effect sequence initiated by the controller but the reciprocal control of each by the other,

This reciprocal communication and control "dialogue" may be verbal or mediated in some way but as long as it continues we can think of the two persons as coupled together to form a new system which develops its own 
characteristic behaviour. Whether or not this resembles the intended outcome of either controlling subsystem is problematical.

Let me illustrate. If you were asked by someone to explain or teach something to him, what would you do? Would you establish performance objective, devise a special sequence of statements to make to him, or insist on special audiovisual displays? What would you look for in order to infer that he understands you?

I conjecture that you might ask him to explain, evaluate or use the relevant knowledge or perhaps to criticize it. You might ask if he has any questions. I doubt if your conversation would be punctuated by multiple choice questions or monosyllabilic responses. I suspect you would probe for evidence of his grasping related concepts or principles. In short, you would function as a supportive conversational system, building on this student's strengths, clarifying misconceptions and linking it into a rich, intricately connected conceptual structure. In the end, both you and the learner have learned something about one another and the subject.

Why do designers of so-called interactive video/CAL systems seldom address these fundamental issues? They proceed instead to present more and more information based on an exceedingly crude inference system that seldom constructs a model of the learner's understanding or permits dialogue. "Right" and "wrong" responses often determine what happens next. But knowledge is more than information. It is complex, relativistic and open to interpretation.

The act of reaching a shared understanding involves agreement (e.g., on explanations, derivations) that one's perception of what the other is saying is an adequate representation of one's own concepts. In short, the structure of knowledge represented by the subject matter expert (as presented through verbal or other media) appears to be congruent with the learner's knowledge structure insofar as they both can perform similar operations of derivation, explanation, identification of counter-examples, application, etc. This dialogue demonstrates reciprocal control by two yoked systems. Such reproducible conceptual representations may be called understanding; a sequence of understandings defines a conversation or, in an educational context, a tutorial ( $c$. Pask, 1976). Our computer-based tutoring systems have yet to achieve this level of dialogue but eventually may approximate it (Mitchell, 1988).

Now consider the possibility that an instructional system is intended to control or regulate the (educational) behaviour of a large number of students simultaneously. One model case is the teacher in a classroom discussion with 25 or more students, each of whom may attempt to control the behaviour of others (as well as themselves). Except under very special circumstances the teacher cannot control the verbal, not to mention the internal behaviour of her students; each responds to others as well as to internal factors. Another model case is the provincial education system which stipulates a set of intended learning outcomes for all students in a particular age group, regardless of individual differences in general or specific knowledge, motivation, etc. and heedless of differences in teachers, learning resources, etc. The typical ap- 
proach to instructional control is to restrict the student's alternatives (rather than to enhance his possibilities).

Even more compelling is the implication of control systems theory that there are fundamental organizing principles in living systems and organizations whereby the observed behaviour is simply the process by which these systems control their sensory input. In other words, the purpose of a system's action is to control the state of its perceived world. This also has some interesting implications for the actions of the researcher as observing system and we must recognize that the observing and the observed system interact; there can be no objective observer.

\section{The Cybernetic Systems Age}

The complexity of inter-related systems with many feedback loops requires us to develop new tools to cope with them. Some relevant tools appear to exist within the trans-disciplinary domain of cybernetics and general systems research.

One of the most impressive aspects of conferences about cybernetics and general systems research is that experts from disciplines as diverse as anthropology and economics, engineering and family therapy, medicine and psychology, natural science and philosophy not only share a meta-discipline that amplifies and transcends their own speciality but also "feel no compunction in tackling the most challenging and vital problems of the day. . believing sincerely that they have, in cybernetics, a powerful inter-disciplinary weapon for solving the most baffling social, economic, and political problems of civilization (Robinson \& Knight, 1972, p. 2).

What is the most important attribute of their approach? Central to the cybernetic or systemic approach is that it considers the total system, with all its interacting elements, as one inseparable organism. This holistic approach represents a paradigm shift from the reductionistic approach which we have inherited from the logical positivist movement. Though the holistic perspective has a strong intellectual background, the word "holism" was invented only in 1925 by Smuts who wrote, "Instead of the animistic, or the mechanistic, or the mathematical universe, we see the genetic, organic holistic universe" (Smuts, 1925).

Synthetic thinking is needed (in addition to analytic) to explain or understand system behaviour. A system is essentially an observer's model which attempts to link a set ofinter-related entities or their attributes into a coherent pattern, one that is perceived to cohere and to be distinct from other entities. This model can be physical, mathematical, verbal or procedural. And the system represented may be physical or conceptual. Indeed it could be argued that all models are fundamentally conceptual and that epistemological issues (e.g., What shall count as information? How can knowledge be represented most usefully?) are central.

The performance of a system as a whole is different from the performance of all its parts. As Gharajedaghi and Ackoff point out, is a whole that 
cannot be divided into independent parts; the effects of the behaviour of the parts on the whole depend on the behaviour of other parts. Therefore, the essential properties of a system are lost when it is taken apart. . . and the parts themselves lose their essential properties" (1985, p. 23). Thus analysis cannot lead to understanding of the system as a whole.

Analysis is very useful for revealing its structure, how it works, but not why it works. Systemic thinking is needed to understand why the system functions as it does. Such synthetic thinking means that we must conceptualize a system as part of one or more larger systems. This calls for seeking understanding of the larger system which, in turn, may be explained in terms of its function in yet another system. This expansionist approach, in contradistinction to the reductionist approach, assumes that ultimate understanding can be approached but that it flows from larger systems to smaller rather than the reverse. Obviously, environmental problems frequently are involved. So are systems that may be called purposeful and human.

Wiener's (1948) use of "cybernetics" to denote the science of control and communications in the animal and the machine, can be restated to omit communications because communications is simply the vehicle for control. Moreover we have seen that regulation may be a less offensive and misleading word. Thus cybernetics is concerned with regulation (i.e., the achievement of goals and objectives of some entity>. As Robinson and Knight (1972) conclude "the central problem remains optimization of the organization and operation; of the organism itself to maximize achievement of its goals and objectives" ( $p$. 5). Moreover, "Any lack of understanding of the nature of this total systems approach results in focus on individual parts of the whole, inability to find much new in cybernetics, and skepticism that cybernetics can add anything worthwhile (p. 5).

In considering the total system, with interacting systems and subsystems, as one inseparable organism, cyberneticians deny the validity (for a complete solution) of optimizing a component subsystem separately. "The approach insists that the analysis be comprehensive and simultaneous. Thus, it considers the total organism ... maximizing achievement of its goals and objectives in its total environment" (Robinson \& Knight, 1972, p. 5). But how do we manage this at the level of society, a university or even a class?

Cybernetics therefore makes possible, explanations of goal-seeking behav-

whether in the human or in organizations. It also permits us to investigate how it is that successful complex systems regulate themselves, in the hope that we may discover principles that can be generalized (cf. Beer, 1986). Equally important for educational technology, we can investigate cybernetic systems with a view to finding out what people or computers are good at and what they are not, thus learning more about how to design expert systems or automated teaching/learning aids.

Can We Redesign Societal Cybernetic Systems for Education?

Our problem is not to portray ideal states of man in the manner of Plato's 
Republic. The best we can hope for, I suspect, is to find out how to regulate a system, in which we are interested, by holding it within its natural boundaries. That is, by monitoring the system's own changes of state as it responds automatically to environmental disturbances, we may be able to control it. On the other hand, if we try to monitor environmental changes we shall fail. Thus the input-output model is obsolescent.

As for our own organizations, Warfield offers this conclusion, "What is needed is the redesign of the decision-making, consensus-building machinery itself, deliberately and carefully employing cybernetic system principles and practices" (1985, p. 80). To do so requires that we design self-correcting cybernetic feedback loops into the structure itself if we wish to produce or manage a viable system (i.e., one that will survive). Beer (1986) offers a model.

Finally we need to recognize that the Conant-Ashby theorem states that the controlling system has to have (e.g., to contain or simulate) a model of the controlled system in order to be able to exert any regulatory control. In the context of educational technology, we must be able to have workable model of our students, the organizations within which we work (or install our solutions), indeed, of our society within the global community. This is a mind-boggling task and our collective failure to do it is one of the reasons for the fatal illness of educational technology.

Perhaps some of us are predisposed to accepting a cybernetic or systemic world view; others may not be. But if educational technology is to become a viable enterprise, I think we will need a massive shift in this direction. Are we prepared? How can any of us acquire this new paradigm if we are not already part of it?

\section{Interaction Within and Between Complex Systems}

Beckwith's insistence on a systemic perspective is not misplaced even though his optimism may be. When dealing with systems as complex as human systems at a global, or even an institutional level we must recognize and cope with the fact that everything interacts with everything else (at least in principle), thus invalidating the traditional analysis and reduction ofproblems into isolated subproblems. This is not meant to be a banal statement.

C. West Churchman, a philosopher of science, expressed it thus:

When we are dealing with systems as complex as human ones, we need to consider: That everythinginteracts with everything else, thus invalidating the traditional reduction of problems into separate subproblems;

That the observer cannot be objective, thus necessitating the development and utilization of an observer-inclusive epistemology;

That ethical and aesthetic variables must be explicitly and effectively integrated into the analysis, design, and decision-making process;

That use of only quantitative data and model-based modes of inquiry is not satisfactory in analyzing and designing human systems; and

That current cross-cultural and culturally specific measures of performance are semantically impoverished. 
In order to develop inquiring systems which will produce results that help to improve the human condition, new approaches, rather than mere extensions and refinements of old ones, are needed. We are convinced such approaches are now available and should be applied to the urgent problems we face (Churchman, n. d.).

Restated, we have to recognize that we cannot describe (e.g., in a mathematical model) any system whose behaviour we wish to regulate because the value of each component's contribution to the overall performance is a function of the current and past activities of all other components as well as of other systems in the environment. If we alter only one factor (or even several) to which the system responds, we may not be able to predict or regulate the outcome. At the cognitive level, virtually every concept is related to others which, in turn, are linked more. But these may be influenced by quite unrelated events.

To illustrate, the academic performance of students may be influenced not only be what the educational technologist does but also by many other factors, (e.g., their genetic endowment, early nutrition and environmental stimulation, previous exposure to information and opportunities to learn and solve problems, psychological stress at home or with peers, blood sugar level, TV viewing, whether or not the nation is at war or experiencing a depression, perception of the subject matter and fellow students - or even of school itself - study skills and decision to select and deploy them, proximity of exams, current events in the community, or the presence and arrangement of specific textual and pictorial messages embedded in salient media). Can we develop an explanatory model to portray this?

To complicate matters, control system theory (Powers, 1973) shows that even when we can relate observed behaviour to observed stimuli, we must expect to be wrong most of the time! Yet our dominant research paradigm (and that of psychology) shows no sign of change.

\section{CONTROL THEORY ANEW PARADIGM FOR BEHAVIOURAL RESEARCH}

Suppose that a visiting scientist from Mars observes an earthling driving a car and decides to investigate the relationship between driving behaviour and the complex pattern of stimuli coming from a twisting, hilly road that is subject to gusts of wind and snow. Suppose moreover, that with his sophisticated methods he found that the stimulus pattern predicted the rate and amount of angular rotation of the steering wheel. Would you be comfortable with this as an explanation of driving behaviour? Or would you, as the driver, insist that in fact it was your intention to drive in the centre of your lane and, because you were successful at it, the visiting scientist failed to notice that there was no deviation of the car's position from this reference trajectory. And if we accept your operational definition of driving behaviour, controlling the 
perceived deviation from the centre of the lane, then we should expect no relationship between this essentially unobservable behaviour and the complex pattern of stimuli.

What is controlled then is controlled only because it is detected by a control system, compared with a goal or reference, and affected by compensatory behaviour based on the perceived discrepancy, Thus a control system controls only its own sensory representation. In this case the control system is controlling an internal representation of the position of the moving vehicle. But note that what is controlled is defined strictly by the behaving system's perception and sensory representation; it may or may not be identifiable as an entity in the external milieu (cf. Powers, 1973). Therefore it may not be identifiable by an observer, especially if it is a perceived discrepancy.

As Powers shows, "In general an observer will not. . . . be able to see what a control system is controlling. Rather, he will see an environment composed of various levels of perceptual objects reflecting his own perceptual organization" and point of view (p. 233). What will he observe? "He will see events taking place, including those he causes, and he will see the behaving organism acting to cause changes in the environment and (his) relationship to the environment. The organism's activities will cause many changes the observer can notice, but what is controlled will only occasionally prove to be identical" with any of them (p. 233).

\section{COMPLEXITY OF GOALS AND NORMS}

To complicate matters for the observer (alias researcher or instructor), human behaviour is not confined to one controlled quantity nor is a fixed reference level the norm. A person, indeed any system, can have multiple objectives and variable reference levels, changing from one to another without warning.

Education, according to philosophers, is concerned with initiating students into instrumentally and intrinsically worthwhile activities. Embedded in this statement is the hint of a narrowly interpreted means-ends concept that seems to permeate educational technology. Let me explain.

We usually think of an end or objective as apositivelyvalued outcome likely to result from some means selected with the intention of producing it. In educational technology the value of a means (e.g., teaching/learningmethod A or B) generally is equated with the probability of its producing an end. Criteria for selection are based on instrumental and cost/benefit decisions. On the other hand, the value of an end is taken to be intrinsic, rather than instrumental. Thus completing my degree may be an end and an educational technologist may use instrumental, extrinsic means to help me to achieve it. But for me, being graduated may be a means to a new job or higher income. And for the educational technologist, the selection of means may be related to personal ends (i.e., intrinsic value for him). In short, every end is also a means and vice 
versa; they are relative concepts.

Note that preferences amongst means may not be based on efficiency but on intrinsic values of the educational technologist. Equally this is true of the student. Each may select means because they are satisfying. Now what if there exists a persistent preference for a particular kind of activity? (Anyone familiar with video game players has seen such behaviour.) Psychologists refer to these as traits. Half a century ago, Gordon Allport identified nearly 18,000 traits. So it is apparent that we can expect to find an exceedingly high variety of ends in any observation of human behaviour except where nearly all of them are eliminated by virtue of the artificial environment of an experiment.

A final note. If we accept that every consequence to some activity is in turn -a means to additional consequences, continuingto some ultimate consequence, then we might find an end that is intrinsically worthwhile. This is essentially a theoretical definition of an ideal. But it is likely that there are many routes to a given ideal and, equally, that a given means-ends activity could eventually be linked to more than one ideal. Given the complexity of human traits and the possibility of many ideals, it is no wonder that observers have considerable difficulty making sense of empirical observations of students' behaviour.

Thus the concept of behaviour as a feedback control process organized around one's perceptions has to be extended to include those perceptions that pertain to ends and means thought likely to maintain one's ideals.

How do we deal with the behaviour of a learner, whether in the laboratory or in the classroom? Are we to conclude that what is observed may not count, that the learner is behaving to reduce a discrepancy perceived by him -not by us-to exist between his current state and some desired state? If so, then it may be incumbent upon educational technologists not merely to have educational (or, more narrowly, behavioural) objectives but to attempt to share responsibility for these with the learner as a control system. More importantly, if we can ascertain the learner's objective we may be able to adapt our instructional activities to support him or her. Truly individualized instruction now might be possible.

\section{SELF-REGULATION FOR SELF-INSTRUCTION}

A closed chain of causal relationships may characterize the learner who is actively studying some subject. Control theory suggests that the learner's behaviour (of attending to and interacting with images and semantic information that may be perceived in the external or internal reference. Any discrepancy "produces" further behaviour intended to reduce this discrepancy, either by re-structuring knowledge and images or by altering the goal. Such control cycles tend to continue until a limiting resource (e.g., time) is used up.

A profound insight reveals the most powerful aspect of feedback: the organism actually "causes" its own behaviour. Sometimes it does so in an environment designed to promote such learning, but educational technology lacks the sophistication needed to develop them. Moreover, the absence of 
universal reinforcers in educational settings underscores the observation (of such investigators as Kelly, Rogers or Snygg \& Combs) that behaviour is a function of individuals' personal frame of reference, their perception of themselves and their environment and the meanings they attribute to them. In cybernetic terms, the person's behaviour controls their perception in relation to their intentions.

\section{Tutoring}

Thus tutoring educational technology can shift from an input-output model to a control theory model dominated by feedback which the observing system uses to control its own behaviour and thereby to attempt to control the behaviour of another system. Note that feedback monitors goal-directed behaviour (i.e., the system begins with some desired state or goal which is compared with its perception of the actual state during or following a behaviour episode). In effect, environment is what the receptors and brain perceive (i.e., an internal representation). Not only objects and events, but also symbols and relations may be represented by these internal models. Internal events probably are represented in the same way and we assume that behaviour (overt or covert) acts on the inner as well as the outer environment.

Powers (1973) demonstrated that what we control is our own input; our behaviour is the means of control and the purpose of our students' action is to control their internal models of the perceived world. This is a powerful insight for educational technologists to exploit. It opens the door not only to design of intelligent CAL but also to the design of new organizational structures for education.

In a tutorial conversation, two cybernetic systems become coupled (until a resource, e.g., time or attention, is used up) to form a new interacting system in which each begins with goals that it attempts to satisfy by monitoring the effects of its own behaviour on the other. Similarly, an adaptive equilibrium occurs between a nation and its education system.

Instead of the input-output model, educational technology could conclude that behaviour is not so much a function of the environmental input as of a selfconscious "I" of each person in interplay not with his environment per se but with his perceptual model of that environment. The would-be regulator of all this, a human or an intelligent CAL system, mirrors the same process; the instructional system must have minimally a model of the subject matter, a model of the student's knowledge and conceptual style, and a model of communications and control strategies to respond to the student's behaviour ( Mitchell, 1982).

What are the implications for educational technology if it's to be rejuvenated? If one's perceptual field determines his behaviour, it seems reasonable to conclude that educational technology has two options. We can continue to implement schemes that limit opportunities for individual differences, developing representations of knowledge that omit much of the richness of a subject in their emphasis on achievement of specifiable objectives in a limited time. Or 
we can recognize individual differences and attempt to promote the optimal development of each person, providing opportunities to extend the selfregulatory capacity of the person both within a subject domain and in general.

To do this, the regulatory system itself will need training. Thus each person, and educational technologists, must learn how to express models of their own activities that have sufficient alternative courses of action from which to choose. Once again I wonder if educational technology has the capacity to do this.

A cybernetic model of the learner, based on Stafford Beer's (1982; 1983; 1984) pioneering work, may prove useful. This model is consistent with research in psychology and education, but begins with a different perspective. At its heart is a perceptual field or set of relationships which determines that this is "Oneself." Beer identifies the intrinsic regulatory mechanism that holds everything together, maintaining one's identity, and suggests that Education should enhance the regulatory variety of each person rather than delimit it (as often occurs). This injunction applies equally at the level of the person and society. Therefore, it may have resuscitating powers for educational technology.

\section{ONESELF, SELF - CONTROL AND THE ENHANCEMENT OF HUMAN POTENTIAL}

If the purpose of one's action is to control the perceived world, a cybernetic model of oneself as learner deserves scrutiny. At its heart is a perceptual field or set of relationships which determines that this is "oneself." Think of a human being not as mind, body, spirit or social unit but as "an entire and interactive system." Oneself is an exceedingly complex, probabilistic system that maintains stability and integrity by virtue of an organizing principle, a set of relationships which determine that this is Oneself, not another self. Beer labels the intrinsic regulator which holds invariant the set of internal relationships that maintains the identity of Oneself, cybercyte.

\section{Goals and Their Achievement}

The self-regulatory capacity of the body seems automatic but what if one aspires to be different (e.g., run amarathon, read 5,000 words per minute, solve a complex problem)? As Beer shows, such pursuits require extending the selfregulatory capacity of both body and mind (i.e., of the cybercyte). Thus I may have the potential to run a marathon or to read at 5,000 wpm or to solve that problem, but I lack the regulatory model required. If Oneself sets goals and aspires to achieve them, then Oneselfmust change one's model ofoneself. Why? Because things one is only potentially capable of doing are not initially included in one's regulatory model. There is a spectrum of options from which to choose (e.g., actions, models, beliefs and aspirations).

Recall that the purpose of human action is to control the perceived world 
by comparing this model with an internal model of a desired end-state. It is essential therefore, that the person (whether learner, researcher, planner or educational technologist) establish a goal-state, believe it can be achieved and will be achieved, and visualize oneself already in the goal state - and then to act accordingly. At this point the regulatory system should respond to perceived deviations from that goal.

Beer's concept of selfhood thus advocates self-improvement - and, by extension, education and societal improvement - based on the existence of autonomous regulatory mechanisms that permit self-control. However, the rules which govern the effectiveness of this self-control require the regulatory process to generate new states and detect and store patterns that can reduce discrepancies. Another principle is that "the recognized self exists within a potential self, the realization of which constitutes its fulfillment" (Beer, 1982, p. 20).

Beer (1984) has tested this model at various recursive levels of selfhood within the context of corporations, society and religion. Surely these principles both address the enhancement of human potential and lie at the core of learning and therefore educational technology. As Beer suggests, Education should enhance the student's regulatory capacity rather than delimit it. But educational technology traditionally has restricted students'regulatory capacity -And our own.

\section{THE DEATH OF EDUCATIONAL TECHNOLOGY}

The preceding discussion of our self-regulatory capacity is central to my analysis of why educational technology cannot escape Beckwith's traps and the major reason for its demise.

Despite Beckwith's (and others') visions of educational technology's potential, the field itself is not a cybercyte and cannot have goals. Individuals can; so can organizations that are established for that purpose. But despite the existence of professional associations, there is no organizing principle that binds and regulates the research, practice and theory development which we identify as educational technology.

Therefore, Beckwith's insistence that educational technology transform itself is misplaced. We who think of ourselves as educational technologists may choose to transform ourselves and even attempt to transform others (e.g., students or colleagues). But even then we may need assistance, perhaps of a kind that does not now exist. We know little of control theory's regulatory models and how to alter the self-regulatory capacity of ourselves or others. Research is needed but who is capable of carrying it out? It may even be that this is one of the most crucial areas for instructional design if we wish to enhance human potential for learning how the world works and how to get along in it.

Graduate programmes in educational technology, too, need to be able to 
communicate relevant insights and research findings to students and, through continuing education and publications, to others. But how can they communicate what they know little about, especially when so many courses address tactical issues at the level of instructional design and media production? Can graduate programmes be transformed along the lines suggested by Beckwith or any other way?

At the same time we function in collaboration with other systems whose perception of educational technology regulates their interaction with us. Do they perceive us to be competent?

\section{NEEDED: ARE-ORIENTATION OF FOCUS}

What stands in our way? Walt Kelly, the creator of the comic strip Pogo, had the main character say: We have met the enemy and he is us."

Educational technology had a short life. By the 70's it had gained academic respectability and widespread acceptance in training circles. As with another new, transdisciplinary field, operational research, "Survival, stability and respectability took precedence over development" (Ackoff, 1979, p. 242). And following Ackoff, I, too, hold academic educational technology and the relevant professional societies responsible for the decline and fall of educational technology. I hasten to point out that I have been involved in both and therefore share this responsibility.

Consider'for a moment what educational technology has contributed to ameliorating existing messes.

Which educational technologists or educational technology programmes have attempted to solve these common problems? (I omit the more complicating systemic implications here.) Reports abound of illiterate and innumerate students graduating from high school. Half the world's children do not go to school. One third of the adult population in the USA (and nearly as many in Canada) is functionally illiterate. Most schools teach children to use computers but not to touch-type so that they can use them more efficiently. Neither teachers nor schools nor ministries of education insist on improved methods of teaching and learning, to say nothing of radical transformation of the curriculum. Our socioeconomic future will require a massive shift in education (and training) just for survival. Atypical educational technology course differs little (except in content) from other courses on campus.

Are educational technology professors or research students tackling such problems? What are the burning issues in educational technology graduate programmes?

Suppose a school of educational technology to be a system organized to produce practitioners for this field. If we were to analyze such a professional school using control theory, what might it look like? Recall that in ordinary behavioural situations the controlled quantity is not immediately obvious and that in a system that operates with the complexity and time span of a graduate 
programme we can expect a very large number of intended outcomes or reference trajectories. Some of these might even be considered to be ideals. Moreover the professional school may attempt simultaneously to contribute to several related goals: to improve society (though its graduates' efforts to improve education); to improve educational systems; to help individuals to increase their knowledge and understanding; to excite in their students a desire and ability to learn and to solve problems.

To the external observer, all that is obvious is the relationship between various "disturbances" applied to the learners and some output of their reorganizing systems. Clearly we should expect to see a shared vision (amongst faculty if not students) of what is desirable but reports from several such programmes suggest that this is not always the case. Then, too, we might expect some creative approaches to the problems of teaching and learning.

For instance, one might test the hypothesis that, "An educational system should (1) facilitate students' learning what they want and need to learn, (2) enable them to learn how to learn more efficiently, (3) motivate them to want to learn" (Gharajedaghi \& Ackoff, 1985, p. 24). One approach may be to assume that the best way to understand a system is to design it (or at least a model of it). To do so, students will need to learn how to solve problems, how to identify what they do not know, how to acquire what they need to know, how to use what they know.

Gharajedaghi and Ackoff suggest a radical departure from standard course-based graduate programmes: their principal instruments are learning cells and research cells which integrate faculty members and students who work jointly to integrate and extend theoretical themes and to design systems or to work on general theoretical, conceptual or methodological problems related to practical problems. The fundamental assumption is that graduate students do not need to be taught but may need guides and mentors. Such an approach clearly permits, indeed encourages, a systemic approach to identifying solving problems. Are we, in educational technology, willing to design radically different approaches to our curriculum and instruction system?

Though I may be mistaken, I think it is fair to say that most educational technology courses are taught by faculty members who have never, or hardly, practiced as educational technologists, except for occasional consulting. They - more accurately, we - and our students are textbook-bound and use the language but not the experiences of dealing with real educational problems, whether we consider complex design problems or simple concepts. By real educational problems I refer not to needs analysis or product development for corporate training (which may indeed be important to the company) but to fundamental problems such as illiteracy, innumeracy, intolerance or lack of caring. To illustrate, I am struck by the blind faith which most of my students have placed in textbook definitions of central concepts, including, for example, "learning." I refer to books which repeat the silly statement that learning is a relatively permanent change in behaviour (as if behaviour of a complex organism is confined to what the observer noted, and - moreover - remains 
static after learning, thus prohibiting further learning). When asked about their own learning experiences such students invariably discuss the concept from a very different perspective, one that is conceptually more useful and defensible. I detect a similar withholding of common sense too frequently in journal articles and textbooks. Something is wrong.

Perhaps because of our being trapped in a state of emulation of an out-ofdate model of science borrowed from psychology, our journals and professional meetings fail, too, to come to grips with very real educational problems. Who is writing (in the educational technology literature) about the messes which we find all about us in the vast domain of education and training?

Is there any hope? Where can we go from here? I am tempted to liken our situation to that of the traveller who asked a farmer how to get to his destination; the farmer replied, "If I were you, I wouldn't start from here." But where can we start from?

\section{FUTURE PLANNING}

Some of us in this disparate field have attempted to act and write as if it were possible to predict future behaviour of a system if only we knew all the cause-effect relations that apply to it. Then, according to this viewpoint, we can design, produce and install some instructional system or materials in such a way as to produce the intended behaviour. Aside from the lack of insight into control system theory which this paradigm reflects, it also fails to take into account the fundamental fact that we operate within constraints that limit our choice just as our clients' choices are limited. Perhaps the most constraining of all is the system within which we function as critical components.

For those in academic educational technology, George Grant warns, We are unable seriously to judge the university without judging its essence, the curriculum; but since we are educated in terms of that curriculum it is guaranteed that most of us will judge it as good. The criteria by which we could judge it as inadequate in principle can only be reached by those who through some chance have moved outside society. . (but then) one's criticisms will not be taken seriously" (Grant, 1968, p. 67). Surely it is this curriculum which has schooled us to believe that certain kinds of theses, publications or papers are somehow more acceptable (albeit to promotion and tenure committees) than others. Research productivity is an ambiguous concept. What counts as research?

The research required to ameliorate some of the pressing educational messes will take many years with little to show for it. What university would give tenure to the modern equivalent of the young Isaac Newton? "To arrive at the simplest truth, as Newton knew and practised, requires years of contemplation. Not activity. Not reasoning. Not calculating. Not busy behaviour of any kind. Not reading. Not talking. Not making an effort. Not thinking. Simply bearing in mind what one needs to know (Brown, 1969, p. 110). For tackling 
complex educational problems such "bearing in mind" certainly is consistent with control system theory even if it is not with contemporary education or our universities. I suspect that this applies to training departments also.

How can those of us who prepare future educational technologists do what is necessary to support these learners in more sustained groping, exploration, synthesis and evaluation as part of their attempts to identify and solve important educational problems? What do we need to contemplate ourselves in order to provide such support? Is research and development in the area of intelligent tutoring systems a useful direction or a dead end? How can we even identify what we need to know so that we may bear it in mind?And how to help our students to do likewise?

One thing is clear to this observer; the corpse called educational technology appears to have died because it lacked a cybernetic systemic paradigm and an organizing principle to give it life as a viable system dedicated to improving education. And even though this field cannot itself easily be a viable system it can contain many viable systems which could even cohere to form such a metasystem. One such component system could be you; I could be another. If we all work together we may just be able to save educational technology and thereby education. But we shall all have to struggle with our regulatory systems. This will require allocation of scarce resources to do the job resources such as care, creativity, commitment and love. Perhaps these are the only assets educational technology has left.

\section{CONCLUSION}

We may be able to revive the corpse of educational technology but not without a radical transformation in a number of inter-related domains: our professional associations; our graduate programmes preparing future practitioners; our schools, colleges, universities and ministries of education; our media of mass communication; our governments; our corporations; our society and - most important - ourselves.

Albert Rosenfeld expressed our educational need thus: "In any planning of society, the structure and function of educational institutions (with education soon to encompass a lifetime) will be at the heart of it; and we are less likely to go wrong in our choices if we keep in mind what it is all to be designed for: the whole human being and his fulfillment in a regulated but free society."

'The educational establishment's major challenge will be to turn out people of high quality; people capable of constantly improving the quality of their own lives and interested in improving the lives of others; people who possess the necessary technical know-how, intellectual prowess, sensory awareness, personal and social responsibility to face cheerfully the unending ambiguities of the new age; people who are incapable ofbestiality toward their fellow men, who have no use for personal power unless it offers an opportunity to enhance the quality of life on earth for all mankind" (Rosenfeld, 1969, p. 311312 ). 
To this I would add that these paragons will need a solid foundation in cybernetics and system thinking as well as in the relevant design sciences. Such educational engineers are likely to become very valuable members of society - if we are able to help prepare them.

If our graduate programmes in educational technology, inter alia, can turn out such men and women then we shall realize Kenneth Richmond's prediction (that this will become the central humane discipline of the future) and Beckwith's dream that we will help "to create health, ideal space and peace." The last reported resurrection required only three days. How long will it take to resuscitate and transform educational technology?

\section{REFERENCES}

Ackoff, R. L. (1979). The Future of operational research is past. Journal of the Operational Research Society, 30 (2), 93-1 04.

Beckwith, D. (1988). The future of educational technology. Canadian Journal of Educational Communication, 17(1), 3-20.

Beer, S. (1986). Diagnosing the system for organizations. Toronto, ON: Wiley.

Beer, S. (1982). Man in a garrulous silence. Unpublished manuscript.

Beer, S. (1983). The will of the people. Journal of the Operational Research Society, 34(8), 797-810.

Beer, S. (1984). The cybernetics of selfhood. Plenary address to Seventh European Meeting on Cybernetics and Systems Research, Vienna.

Brown, G. S. (1969). Laws of form. London: George Allen and Unwin

Grant, George (1968). The university curriculum. In H. Adelman \& D. Lee (Ed.) The university game. Toronto: Anansi.

Gharajedaghi, J., \&Ackoff, R. L. (1985). Toward systemic education of systems scientists. Systems Research, 2(1), 21-27.

Mitchell, P. D. (1970). Educational technology: Panacea of placebo?, Aspects of Educational Technology IV. London: Pitman.

Mitchell, P. D. (1973). The professional development of educational technologists, Aspects of Educational Technology V. London: Pitman.

Mitchell, P. D. (1975). The discernible educational technologist. Programmed Learning and Educational Technology, 12(5), 306-325.

Mitchell, P. D. (1982). Representation of knowledge in CAL courseware, Computers \& Education, 6, 61-66.

Mitchell, P. D. (1988, June). Evaluation of understanding in intelligent interactive systems: An application of conversation theory. Paper presented to the Fourth Conference on Development of Effective Interactive Instruction materials, Stanford, CT.

Pask, G. (1976). Conversation theory. Amsterdam: Elsevier.

Powers, W. T. (1973). Behaviour: The control of perception. New York, NY:

Richmond, W. K. (1967). The educational industry. Methuen, London. 
Robinson, H.W., \& Knight, D.E. (Eds.) (1972). Cybernetics, artificial intelligence and biology. New York: Spartan Books.

Rosenfeld, A. (1969). The second genesis: The coming control of life. Englewood Cliffs, NJ: Prentice Hall.

Schwen, T. M. (1988). An organizational analysis of the future of educational technology. Journal of Instructional Development, 11(3), 21-27.

Smuts, J. C. (1925). Quoted in the supplement to the Oxford English Dictionary, 1971.

Warfield, J. N. (1985). Developing a design culture in higher education: Some laws and principles of design. Proceeding of the 1985 International Conference of the Society for General Systems Research, Vol II, (pp. 725-729). Seaside, CA: Intersystems Publications.

Wiener, N. (1948). Cybernetics. Cambridge, MA: M. I. T. Press.

\section{AUTHOR}

P. David Mitchell is a Professor in the Graduate Programs in Educational Technology at Concordia University, 1455 de Maisonneuve Blvd. W., Montreal, PQ H3G 


\section{Your comment would be}

appreciated!

The previous article represents one professional's perspective on the current state and future prospects of educational communic ation and technology as a field of study and practice.

In a continuing effort to generate dialogue conceming the issues raised by this a uthor - issues that all of us may face sooner or later - we invite you to respond with a publishable reply. Maximum length: 1000 words. Comments must be signed and may be edited for style and length at the disc retion of the Editor. Some comments may not be published.

Send to:

Dr. Robert M. Bemard

Department of Education

Concordia University

1455 de Maisonneuve Blvd. W.

Montreal, PQ

H3G 1M8 


\title{
Modifying the Regulation Processes of Leaming: Two Exploration Training Studies
}

\author{
P. R.J. Simons
}

\begin{abstract}
Abstact Self-regulated leaming is defined as leaming in which a student performs teaching tasks himself. Regulations of leaming (e.g.. orientation, planning, testing, monitoning) are thought to be important both in teacher - and self-regulated leaming. Furthemore, mind orientation and distractions are expected to ham effective learning. Two studies are reported on individual differences in regulationprocesses. Thinking aloud protoc ols of good and weaker perfoming subjects were analyzed as to the number of regulations. mind orientations and distractions and related to test scores (impulsivity, intelligence, concentration, motivation, etc.). Furthemore, students were trained to modify their regulation processes. Training programs consisted of a combination of awareness training and regulation training. The subjects were 10 and 6 students from two secondary schools (for special education), respectively. The results showed some relations between process differences on the one hand and performance on the other hand. There were also influences of task difficulty on the process data. Training appeared to be effective for some of the students only. Transfer effects failed to appear,
\end{abstract}

\section{INTRODUCTION}

\section{Self-regulation Versus Teacher Regulation}

A theoretical framework derived from the theories of Boekaerts (1982), Brown (1980), Gagne (1977), Hettema (1979), Klauer (1985), Kuhl (1983) and Lawson (1984) is the basis of the research reported in this paper. According to this framework, self-regulation of learning is defined as the number and kinds of teaching tasks students perform themselves. Five main teaching tasks (see Figure 2) are discerned: preparing learning; facilitating learning; regulating learning; giving feedback; and judging performance and keeping students concentrated and motivated. Self-regulation, in our opinion, thus pertains to the extent to which one is able to be one's own teacher and to perform the teaching tasks oneself: being able to prepare one's own learning; to take the necessary steps to learn; to regulate learning; to provide for one's own feedback and judgement; and to keep oneself concentrated and motivated.

The most extreme form of self-regulated learning occurs when students perform all of these teaching tasks themselves. Most of the time, however, 
teachers (or their substitutes, for instance books or computers) take care of at least part of these tasks. In essence, there always seems to be a division of tasks. Extension of the responsibility of students for their own learning may in some cases improve learning. Lodewijks (1981) for instance showed that students learning science concepts in a self-chosen sequence performed better then students learning these concepts in a predetermined sequence. Likewise, Van der Sanden (1986) showed that some students (especially the better ones) performed better on a practical construction task without instructions than with detailed and explicit advice from a teacher.

According to these and other studies, improvement of learning might be reached by giving students more opportunities to regulate their own learning. This, however, is problematical in practice. Apart from the students who might profit from these opportunities, there are also students who will perform worse when teacher advice is absent (Lodewijks, 1981; Van der Sanden, 1986). A differentiated system with opportunities for self-regulation for the better performing students and sound advice for the weaker students, however, encounters many practical disadvantages and problems. As was discussed by Larsson (1983) paradoxes of teaching should also be taken into account. Some teachers would like to give students more freedom to learn but do not believe that students are able to handle this freedom. Some students believe that only the teachers should make decisions on learning and seem to hand over all responsibility to the teachers. In our opinion there is only one way out of these and other paradoxes and circularities and that is by training students in selfregulation. One main goal of training programs should be to convince students that they have a responsibility for their own learning and that they can acquire the skills to regulate their learning.

In our conception (see also Hettema, 1979; Lawson, 1984), three levels or perspectives in respect to the teacher and self-regulation tasks should be discerned (see figure 1): (metacognitive) knowledge and conception (for instance knowledge of study strategies; knowing when to use certain strategies; or conceptions of self-regulation); executive control or regulation processes

Figure 1.

Three Perspectives and Their Interrelations

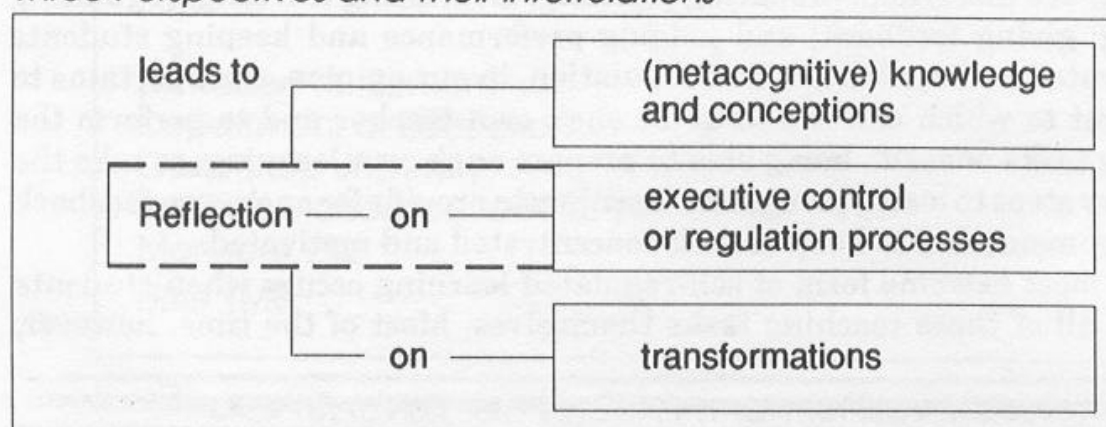


(for instance deciding on a plan, attention-maintenance, monitoring, or repairmechanisms); and transformations or executive skills (for instance paraphrasing, underlining, understanding or reading).

In agreement with Lawson (1984) and recent Russian theories (e.g., Zak, 1980) we assume that metacognitive knowledge arises from reflection (being an executive control process itself) on executive control processes transformations.

\section{Subtasks of Self and Teacher Regulation}

Some of the teaching and self-regulation tasks discussed above have subtasks (see figure 2 on following page): The first task (preparing learning) has subtasks borrowed from Gagne (1977) and Galperin (1969): orientation, planning, gaining attention, promoting self-confidence, informing on goals, recall or previous learning. The second task (facilitating learning) is formulated in accordance with suggestions by Boekaerts (1982) and Klauer (1985). The subtasks are: facilitation of remembering and comprehension, of integration and of problem solving. For the third teaching task we extrapolated from theories of Brown (1980), Hettema (1979), Lawson (1984) and our own research (see Simons and Lodewijks, 1987): monitoring testing and questioning, revision and evaluation. The fourth task (feedback and judgement) comes from Gagne (1977) and the last one (upholding motivation and concentration) has been formulated in agreement with Kuhl's theory on mind orientation and activity orientation. According to this theory, in ideal mental states (action orientation) attention is divided evenly between a) the beginning state; b) the goal state; c) the discrepancy between the present state and the goal state; and d) the path that leads from a to b. In mind orientation, however, attention fixates on one or two of the four components of a fully developed action structure. Kuhl (1983) discerned four kinds of fixations (or four kinds of mind orientation): goal fixation, planning fixation, failure fixation and success fixation. Teachers should be attentive to these. Moreover, they should try to lead students towards an activity orientation in which the goal state to be reached, the present state, the difference between the goal and the present state and the plan that could be used to change the present state into the goal state each get sufficient attention. One aid teachers might use to help students reach these states is goal setting. In self-regulated learning students should be action oriented instead of mind-oriented.

\section{Previous Studies}

In our previous work (see for instance Simons and Lodewijks, 1987) we studied individual differences in the self-regulation of learning, both at the regulation level and at the metacognitive knowledge level of figure 1, emphasizing the preparation and regulation tasks of figure 2 (e.g., orientation, planning, monitoring, testing, revision and evaluation). Furthermore, we tried to change learning performance and

processes of students through a relatively short training program. The training was based on the 
Figure 2.

A Categonization of Teaching and Self-regulation Tasks

Preparing learning

* Orientation on goals, strategies, time, etc.

* Planning of learning (time, anticipation of problems, choice of strategies).

* Gaining attention

* Promoting self-confidence

* Informing students on goals

* Stimulating recall of prerequisite learning

II Facilitating learning

* aimed at remembering and comprehension.

* aimed at integration with other information

* aimed at problem solving

III Regulating learning

* Monitoring

* Testing and questioning

Revision (re-orientation, diagnosing, reflecting, repairing)

* Evaluating learning processes

IV Giving feedback and judging performance

V Upholding concentration and motivation

differences in processes observed during a pretest session. Metacognitive awareness was stressedby letting students reflect on their own way oflearning as well as that of other students. Regulation processes were trained through practice with a set of questions one may ask oneself during learning (e.g., Do I understand this part? What went wrong? Is this in line with the learning goal?) and a set of techniques and skills one may find useful in answering these questions (e.g., paraphrasing, reflection, thinking of new examples, self-testing). For the training program two case histories were written depicting two totally different ways of learning: a passive way and an active way incorporating several self-diagnostic routines and heuristics. Also, a short booklet was written in which the set of questions one may ask oneself during learning was described. Moreover, for each question, suggestions were put forward for 
possible ways to answer these questions. Finally, a set of practice materials (texts, words and problems) was constructed.

The subjects in the study reported in Simons and Lodewijks (1987) were 14 students from the second year of secondary school. Ages ranged from 13 to 15. Three sets of learning tasks were used, consisting of two parallel texts of 900 words on probability, the one introducing principles, problems and examples of chances with replacement, the other dealing with chances without replacement, two sets of 20 French words and their Dutch translations and two parallel problem solving assignments, in which simple probability principles (introduced in a separate text) had to be applied. Following Olshavsky (1976), red dots were put in places in the texts where verbalization was thought to be crucial.

This study revealed some interesting relations between performance and individual differences in regulation processes. In text processing the tuning of self-diagnostics to the learning goal proved to be the most important aspect. In vocabulary learning, however, the amount of self-testing differentiated between good and weaker performing students. In the problem-solving task monitoring, regulation and orientation processes showed up more frequently with better performing subjects. Thus, individual differences in processes correlating with performance were task-dependent. Training proved to be effective for the text processing only. One important and unique outcome of the study was that there was not only a training effect on learning performance, but also on the frequency of occurrence of some of the regulation processes. Especially the number of task relevant self-testings increased as a result of the training. Though many previous studies succeeded in showing an effect of training on performance (for instance Palincsar and Brown, we did not find any other studies showing an effect of training on regulation

One problem in this study concerned assumptions of cause and effect. The quality of regulation processes could cause the effectiveness of task performance. In the case of the text processing data we have reason to believe that this is what happened. Apart from the correlation between performance and processes, there was also a change in the number of testings on understanding (induced by the training program) coinciding with a change in performance. For the other two tasks, however, the causal relation could also be in the reverse direction. Weak performance (for instance caused by low abilities) might cause the occurrence of particular processes, like noticing negative results or continuing planning. Therefore, a distinction between good and bad regulation processes would be helpful, Kuhl's distinction between mind orientation and activity orientation processes might be a solution. Therefore, we decided to extend our categorization scheme with new categories pertaining to mind orientation and distraction.

The main research questions of the present studies were the same as for the previously described one: a) What individual differences in self-regulation occur and which of these related to performance differences? and b) Is there an effect of training on process and performance variables? 


\section{STUDY 1: REGULATION PROCESSES, MIND ORIENTED PROCESSES AND DISTRACTIONS}

In this study, an attempt was made to discern good and bad regulating processes, using Kuhl's theory on mind orientation. The categorization scheme was extended with mind oriented processes (directed to failure or success experience, e.g., "I can't do it" and "I succeeded last time," valuations of the task at hand, e.g., "This is too difficult for me" or "I hate these sums," planning fixation, e.g., "How can I solve this" or goal fixation, e.g., "If only I were ready") and task-irrelevant statements (distractions). Moreover, in order to get an impression of the validity of the process measures, relations with impulsivity, concentration ability, verbal intelligence and motivation were studied. Finally, students from special education were the subjects of this study, because we were afraid that we would not get enough statements in our new categories when employing students from a normal school.

\section{METHOD}

\section{Subjects}

The subjects were 10 boys from a secondary school for special education. They were selected by the school because of their weak concentration abilities. Ages ranged from 12 to 14 years.

\section{Materials}

In this study arithmetic word problems formed the main learning materials. Because of the learning disabilities of the subjects, the tasks used in the previous study could not be used. Arithmetic word problems were chosen because of the difficulties they pose for this kind of pupil (according to the teachers). We wanted to restrict the training to one type of task in order to prevent confusion between strategies for different tasks. In total, 11 word problems like the following constituted the training material: "A train departs at 21:47 hours. Travelling time is 3 hours and 36 minutes. At what time will the train arrive?."Another set of 7 of these story problems formed the pre-test and another 7 were the post-test. Also, both at the pretest session and at the post-test session, transfer tasks were administered: 12 fraction problems like 4/- = 6/9 and 2 problem-solving tasks. These tasks consisted of a description and a drawing of a route to be taken, for instance from school to home. On the way some other things had to be done, like visiting a library, shopping, delivering something to a friend. Several time constraints as to how long a certain route takes, how long you need for a task or when something had to be done (e.g., the shop closes at 6 p.m.> form the data to be used. The task of the subject is to find the fastest way home.

The following standardized tests were used: a concentration test (Bourdon-Wiersma), an achievement motivation test (PMT-K), the Matching Famil- 
iar Figures Test (MFFT) and the verbal analogies subtest of an intelligence test (Differential Abilities Test).

During training, subjects learned a heuristic self-regulation strategy to be used when solving arithmetic word problems. The following questions were used as examples: 'What exactly is the question posed?"; "Did I understand everything?"; What calculations are to be made?"; What is the best way to handle this?"; "Where can I start?"; "Why don't I understand this?"; What am I doing?"; "Is this outcome acceptable?"; What mistake did I make?"; "Is there yet another way to solve this problem?".

\section{Procedure}

There were 4 phases in this study In the first individual session (taking 2 hours) the tests were administered. Also, the subjects were trained in thinking aloud, and in using materials comparable to the ones used in later phases.

The second phase consisted of the pretest session (1.5 hours), in which the arithmetic word problems and transfer tasks were administered, subjects thinking aloud all the time.

In the third phase only half of the subjects participated. As a group they were trained during two sessions (4 hours in total). In the first session a group discussion on concentration and self-regulation problems took place (awareness training). Aquestionnaire, measuringreactions to concentration and selfregulation problems, developed in a previous study, was administered individually and the results were discussed in the group. Students were then informed on possible ways to react to concentration and self-regulation problems. Aheuristic self-questioning and answering strategy used in the previous study was then demonstrated by the investigators, solving word problems. During the second session the subjects practiced with this strategy, solving 11 word problems individually. The subjects had to ask themselves the questions they had learned before. Their solution processes were recorded on video. After every word problem the video tape was rewound and the solution processes were shown to the subjects. The investigator and the subject then discussed these in light of the heuristic strategy.

Finally, the individual post-test session took place. All 10 subjects again solved 7 arithmetic word problems and the transfer problems, thinking aloud all the time.

\section{Design}

The design was a pretest-post-test-control group design with random assignment to the two conditions.

\section{Data Analysis}

For each of the three tasks (word problems, fractions and problem solving) two groups were formed: the subjects performing above and below the median. Processes of these groups were compared, using t-tests for independent samples. Where significant differences in variance appeared, separate vari- 
ance estimates were used. Four categories of processes were used: execution (all covert and overt activities transforming states of knowledge or understanding in the direction of the goal states, e.g., reading, writing), control (all statements that refer to activities regulating the executive actions like orientation, planning, self-testing, monitoring, revision and evaluation, e.g., "Oh yes, I understand," "If I read on I will understand it," Will I be tested?", "Therefore I must conclude that she must be younger") (see appendix 1), mindorientated (all statements pointing to fixations on prior successes or failures, planning or goals, see the introduction for examples) and distracted (all statements that were judged task irrelevant, reacting to external or internal stimulations, e.g., "There is a pigeon on the roof," "Tomorrow, I am going to play with my computer").

Product moment correlations were determined between process measures themselves and between process measures and test scores. The differences in regulation processes and in performance on the word problem task as well as the two transfer tasks between the trained and the untrained group, were analyzed by way of analysis of covariance.

\section{Results}

Table 1 (on the following page) presents the mean differences in processing between subjects scoring above and below the medians of the three performance measures. As to the arithmetic word problems no significant differences appeared (t-values of $.60, .08, .94, .56$ respectively). There was a tendency for weaker subjects to utter somewhat more mind-oriented and distracted statements. For the fraction, problems differences showed up as to execution $(t=2.69, \mathrm{df}=8, \mathrm{p}<.05)$ and control $(\mathrm{t}=2.05, \mathrm{df}=8, \mathrm{p}<.10)$. The difference in frequency of mind-orientation and distraction was not significant (t-values of 1.8 and 0.0). For the problem-solving task a similar phenomenon showed up, but now in the reverse direction. Better performing subjects had higher frequencies in execution $(t=-.12, \mathrm{Df}=4.6, \mathrm{p}<.10)$ and control processes $(t=1.0$, $\mathrm{df}=5.1, \mathrm{p}<.10)$ than weaker performing subjects. In all three tasks the mean number of mind-oriented and distracted cognitions was rather low.

Table 2 (on the following page) presents the correlations between the different process categories for the three different tasks. Four of the correlations reached significance, the others were moderately high. The correlations for the mind orientation scores were rather low, possibly because of the low frequencies of occurrence. In Table 3 (see page 38) the correlations are reported between the process measures (word problems only) and the test scores. Significant correlations appeared between mind orientation and intelligence and impulsivity and between distractedness and concentration.

The training program failed to be effective, as can be seen in Tables 4 and 5 (see page 39). None of the analyses of covariance with pretest product and process scores as covariates and post-test product and process scores as dependent variables reached statistical significance. Performance on the word problems did not increase significantly from pre- to post-test, either in the 
Table 1

Mean Frequencies of Processes for good and Weak Performing Subjects on the Three Tasks (SD's in brackets)

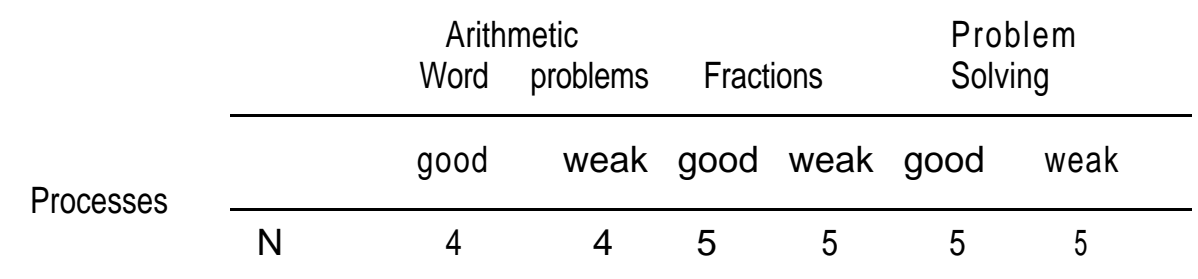

$\begin{array}{llcccccc}\text { Execution } & M & 20.5 & 24.0 & 19.6 & 39.0^{* *} & 17.4 & 5.8^{*} \\ & S D & (9.8) & (6.4) & (6.3) & (14.9) & (11.7) & (3.3)^{* *} \\ \text { Control } & M & 12.8 & 13.3 & 8.6 & 26.0 & 14.6 & 4.5^{\star} \\ & S D & (11.7) & (5.4) & (7.6) & (17.3) & (10.2) & (3.8) \\ \text { Mind- } & M & 5.3 & 8.5 & 4.4 & 11.6 & 1.4 & 0.2 \\ \text { oniented } & S D & (5.1) & (4.7) & (5.6) & (6.9) & (2.5) & (0.5)^{\star *} \\ \text { Distra cted } & M & 1.8 & 2.5 & 3.8 & 3.8 & 4.8 & 0.6 \\ & S D & (1.7) & (2.1) & (4.7) & (4.1) & (6.9) & (0.9)^{* *}\end{array}$

$*=.05<p<10$
$* * \quad P<.05$

Table 2

Correlations Between the Process Measures for the Three Different Tasks $(N=10)$

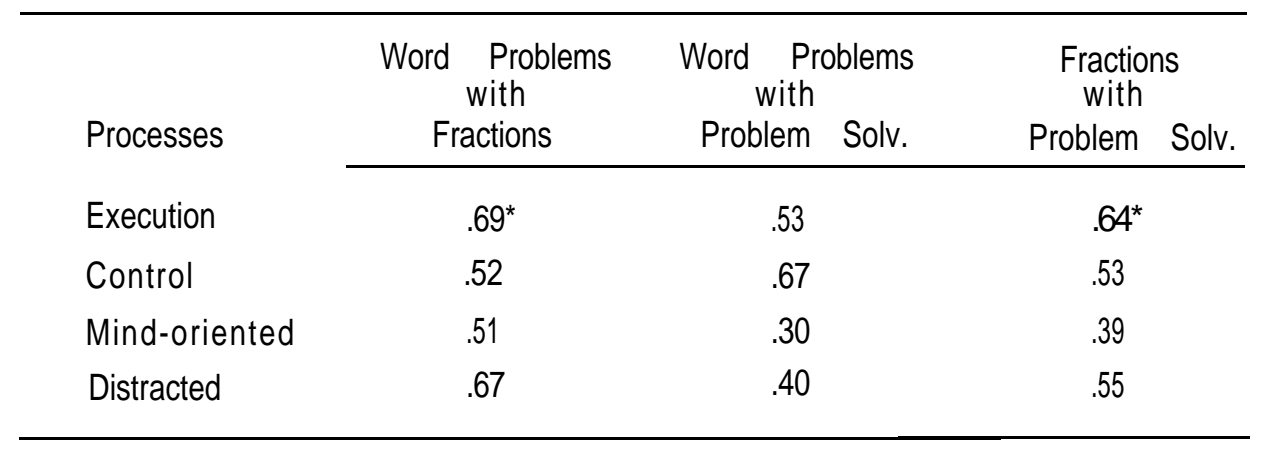


Table 3

Correlations Between the Process Measures for the Word Problems ( $\mathrm{N}=10)$ and Test Scores

\begin{tabular}{|c|c|c|c|c|c|}
\hline Processes & $\begin{array}{l}\text { Ach. } \\
\text { Mot. }\end{array}$ & Fear & Intel. & Concen. & Impuls. \\
\hline Execution & .20 & .60 & .32 & -.48 & .58 \\
\hline Control & .10 & .53 & .33 & -.45 & .43 \\
\hline Mind-oriented & -.55 & .49 & $.63^{*}$ & .12 & $.64^{*}$ \\
\hline Distracted & .44 & .20 & .48 & $-.64^{*}$ & -.15 \\
\hline
\end{tabular}

Ach.Mot $=$ Achievement motivation, Fear $=$ Fear of failure

Intel. = Intelligence (verbal analogies), Concentr. = Concentration, Impuls. = Impulsivity. ${ }^{*} p<.05$.

training group ( $\mathrm{t}=\mathrm{l} . \mathrm{l}, \mathrm{df}=4$, n.s.) or in the control group $(\mathrm{t}=1.5, \mathrm{df}=4$, n.s.). Trends in the process data were in an unintended direction. In the trained group a (non-significant) increase, instead of a decrease, in the mean number of mind-oriented and distracted statements appeared. Since there was no effect on the process and performance data of direct dependent variable (the arithmetic word problems), no transfer to the fractions and problem-solving transfer tasks showed up either, as was to be expected.

\section{STUDY 2: MODIFYING PATTERNS OF MIND ORIENTATION}

In this study an attempt was made to solve some of the problems encountered in the previous one. One problem was the relatively small number of mind-oriented and distracted statements appearing in the protocols. Therefore mind orientation was operationalized in a different way. Kuhl defined the distinction between mind and activity orientation on a molar level. He defined activity orientation as a state of mind in which both the present state, the goal state, the difference between these two and the possible actions get attention from the subject and mind-orientation as a state of mind in which a fixation on one of these four elements occurs. Perhaps a more holistic approach in analyzing protocols should be taken. Instead of registering single mindoriented statements, patterns of statements were sought that might be indicative of mind orientation. There was no separate category for mindoriented verbalizations.

Another problem was the lack of effect of the training program with the 
Table 4

Mean Pre-test and Post-test Results on Word Problems in the Trainea and Untrained Groups (SD's in brackets)

\begin{tabular}{lccccc}
\hline & & $\begin{array}{c}\text { Trained } \\
\text { pretest }\end{array}$ & $\begin{array}{c}\text { Group } \\
\text { post-test }\end{array}$ & \multicolumn{2}{c}{$\begin{array}{c}\text { Control Group } \\
\text { pretest }\end{array}$} \\
Variable & $\mathrm{N}$ & 5 & 5 & 5 & 5 \\
\cline { 2 - 6 } performance & $\mathrm{M}$ & 2.0 & 3.2 & 1.8 & 3.0 \\
on word & $\mathrm{SD}$ & $(1.2)$ & $(1.3)$ & $(2.2)$ & $(2.0)$ \\
problems & & & & & \\
\cline { 2 - 6 } Execution & $\mathrm{M}$ & 21.0 & 24.2 & 25.0 & 21.6 \\
& $\mathrm{SD}$ & $(8.3)$ & $(14.1)$ & $(7.3)$ & $(5.2)$ \\
Control & $\mathrm{M}$ & 12.2 & 12.6 & 15.6 & 13.2 \\
& $\mathrm{SD}$ & $(8.7)$ & $(14.2)$ & $(9.1)$ & $(7.6)$ \\
Mind-oriented & $\mathrm{M}$ & 5.4 & 6.4 & 9.6 & 8.0 \\
& $\mathrm{SD}$ & $(4.4)$ & $(9.0)$ & $(3.8)$ & $(2.6)$ \\
Distracted & $\mathrm{M}$ & 3.8 & 8.8 & 1.6 & 3.8 \\
& & $(2.2)$ & $(6.3)$ & $(1.1)$ & $(3.4)$ \\
\hline & & & & &
\end{tabular}

Table 5

Analyses of Coverance on the Product and Process Measures (word problems only)

\begin{tabular}{lllllll}
\hline $\begin{array}{l}\text { Dependent } \\
\text { Variable }\end{array}$ & Covariate & $\begin{array}{c}\text { MS } \\
\text { covar. }\end{array}$ & $\begin{array}{c}\text { MS } \\
\text { condition }\end{array}$ & $\begin{array}{c}\text { MS } \\
\text { error }\end{array}$ & $\boldsymbol{F}$ & Sig. \\
\hline $\begin{array}{l}\text { word } \\
\text { problems }\end{array}$ & $\begin{array}{l}\text { pre-test } \\
\text { word problems }\end{array}$ & 1.0 & 0.8 & 3.1 & .02 & n.s. \\
$\begin{array}{l}\text { execution } \\
\text { post-test }\end{array}$ & $\begin{array}{l}\text { execution } \\
\text { pretest }\end{array}$ & 108.0 & 46.3 & 113.1 & .41 & n.s. \\
$\begin{array}{l}\text { control } \\
\text { post-test }\end{array}$ & $\begin{array}{l}\text { control } \\
\text { pretest }\end{array}$ & 251.4 & 6.2 & 112.0 & .79 & n.a. \\
$\begin{array}{l}\text { mind- } \\
\text { oriented } \\
\text { post-test }\end{array}$ & $\begin{array}{l}\text { mind- } \\
\text { oriented } \\
\text { pre-test }\end{array}$ & 104.2 & 31.3 & 35.3 & .28 & n.s. \\
$\begin{array}{l}\text { distract. } \\
\text { pro-test }\end{array}$ & $\begin{array}{l}\text { distracted } \\
\text { pretest }\end{array}$ & 6.0 & 65.9 & 28.0 & .17 & \\
\hline
\end{tabular}


children with concentration problems as opposed to the effects found in our previous work with "normal" children. The training program was changed considerably by incorporating new elements like reciprocal teaching procedures (Palincsar \& Brown, 1984), individual learning goals based on protocols collected during a pre-test session and modelling. As a consequence of this, the training took approximately twice as long as in the previous study.

\section{METHOD}

\section{Subjects}

The subjects were 4 boys and 2 girls from a school of special education, selected out of a group of 45 students on the basis of 6 criteria: weak concentration according to the teacher and the school psychologist; impulsivity (MFFT); age (12 years old); low achievement motivation; hightest-anxiety and, sufficient mathematical ability,

\section{Materials}

As in study 1, arithmetic word problems formed the learning task. For the pre-test, 3 word problems were used, the same being done for the post-test. Reading comprehension was used as a transfer measure. Both during the pretest session and the post-test session the subjects studied a text of3 pages, 1000 words (one text on "old times", the other one on "parents evening") and answered open-ended comprehension questions about its contents.

For all subjects individual learning goals were formulated on the basis of their pre-test thinking aloud-protocols. For each individual training session a script was prepared, concretizing how the individual learning goals could be reached. The elements included in the training were: reciprocal teaching procedures, experimenters and students changingroles, modelling, awareness training, direct instruction on regulation mechanisms, prompting, and feedback on regulation processes. In two group sessions $(\mathrm{N}=3)$ students worked together and discussed their regulation processes.

\section{Procedure}

The first session was a pre-test session. It started with an exercise in thinking aloud when solving a word problem. Subjects were taught how to think aloud, and received feedback. After that the three word problems were solved thinking aloud. Duringthe second session, the text was read, again with the subjects thinking aloud. Directly afterwards 8 comprehension questions were answered on the content of the text. The third session took place 10 days later and constituted the first individual training session. In between, the thinking-aloud protocols were typed out and analyzed in order to formulate the individual learning goals. This third session was dedicated to awareness training, following the procedure used in study 1 . The fourth session (2 days later) was an individual session as well. Now three word problems were solved, 
following a reciprocal teaching procedure. Two days later a group session (6 students and 2 experimenters) followed. Regulation processes were modelled by the experimenters. Students were stimulated to work together and to be each other's external monitor. Also, important conclusions from the individual sessions were repeated and discussed. The sixth and seventh sessions again were individual training sessions, the procedure being the same as in the fourth session. Different kinds of word problems were used as training materials. The eighth session was another group session (like session 5). Students now learned the differences between five kinds of word problems. Furthermore, they wrote down what they thought they had learned from the training. The final session was the post-test session, and was identical to the first session.

\section{Design and data analysis}

The design was a pre-test-post-test design. For the categorization of the thinking-aloud protocols, the same categories were used as in the previous studies. This time, however, a detailed analysis was made of the subcategories of the "control" category used in study 1 . These subcategories are defined in appendix 1 .

\section{Results}

There was a significant increase in scores on the arithmetic word problems from the pretest to the post-test $(\mathrm{M}$ pretest $=2.1 \quad(\mathrm{SD}=2.2), \quad M$ pos-ttest $=5.5$ $(\mathrm{SD}=2.4) ; \mathrm{t}=2.50, \mathrm{p}$ (one-tailed) <.05). Transfer to the text comprehension performance, however, did not occur (M pre-test=4.9 ( $\mathrm{SD}=4.9), \quad M$ post-testz4.9 $(\mathrm{SD}=1.7) ; \mathrm{t}=.03$, n.s.).

In Table 6 (on the following page) frequencies of the different processes per subject are presented, both for the pre-test and-for the post-test session. Subjects 2 and 5 increased their number of verbalizations in almost all categories. These were also the 2 subjects who profited the most from the training in terms of performance improvement, Subjects 4 and 6 increased their number of execution, regulation and testing statements. Subject 1 increased the number of execution and testing verbalizations and for subject 3 an increase in the number of orientation and regulation statements could be noticedandadecreasein the number of monitoring statements. These changes did not occur as to the verbalizations during text comprehension, as may be seen from Table 7 (on page 43 ).

In order to find changes in mind and activity orientation, the thinkingaloud protocols were analyzed per word problem and classified according to the 4 kinds of fixations discerned by Kuhl (3 word problems per subject per session). The results are presented in Table 8 (page 44). There was a significant difference in protocol patterns before and after the training (chi-square $=6.9$, $\mathrm{p}<.01$ ). The number ofnegative and positive self-statements was also counted. The number of negative self-statements decreased from pre-test to post-test for 3 subjects during the solution of the word problems and for all subjects during 
Table 6

Frequencies of the Different Processes. Before and After Training (arithmetic word problems)

\begin{tabular}{|c|c|c|c|c|c|c|c|}
\hline \multirow[b]{2}{*}{ Process } & \multirow[t]{2}{*}{$N$} & \multicolumn{2}{|c|}{1} & \multicolumn{2}{|c|}{2} & \multicolumn{2}{|c|}{3} \\
\hline & & $\begin{array}{l}\text { pre- } \\
\text { test }\end{array}$ & $\begin{array}{c}\text { post } \\
\text { test }\end{array}$ & $\begin{array}{l}\text { pre- } \\
\text { test }\end{array}$ & $\begin{array}{l}\text { post } \\
\text { test }\end{array}$ & $\begin{array}{l}\text { pre- } \\
\text { test }\end{array}$ & $\begin{array}{l}\text { post } \\
\text { test }\end{array}$ \\
\hline Execution & & 18 & 32 & 25 & 65 & 58 & 57 \\
\hline Orientation & & 11 & 11 & 7 & 16 & 8 & 12 \\
\hline Monitoring & & 20 & 20 & 11 & 44 & 31 & 21 \\
\hline Regulation & & 13 & 11 & 8 & 30 & 24 & 29 \\
\hline Testing & & 2 & 8 & 2 & 11 & 5 & 1 \\
\hline Diagnosing & & - & 1 & 1 & 1 & . & . \\
\hline Evaluation & & - & - & 1 & - & - & - \\
\hline Distracted & & 4 & 2 & 1 & - & - & - \\
\hline
\end{tabular}

\begin{tabular}{lrrrrrr} 
Subject N & \multicolumn{2}{c}{4} & \multicolumn{2}{c}{5} & \multicolumn{2}{c}{6} \\
\cline { 2 - 7 } Process & $\begin{array}{c}\text { pre- } \\
\text { test }\end{array}$ & $\begin{array}{c}\text { post- } \\
\text { test }\end{array}$ & $\begin{array}{c}\text { pre- } \\
\text { test }\end{array}$ & $\begin{array}{c}\text { post } \\
\text { test }\end{array}$ & $\begin{array}{c}\text { pre- } \\
\text { test }\end{array}$ & $\begin{array}{r}\text { post } \\
\text { test }\end{array}$ \\
\cline { 2 - 7 } Execution & 7 & 23 & 5 & 230 & 1 & 18 \\
Orientation & - & 1 & - & 55 & 5 & 2 \\
Monitoring & - & 4 & 4 & 164 & 10 & 7 \\
Regulation & 4 & 14 & 8 & 93 & 2 & 8 \\
Testing & - & 6 & - & 32 & - & 2 \\
Diagnosing & - & - & - & 7 & - & - \\
Evaluation & - & - & - & 2 & - & - \\
Distracted & - & - & & 5 & - & -
\end{tabular}


Table 7

Frequencies of Different Processes, Before and After Training (text comprehension)

\begin{tabular}{|c|c|c|c|c|c|c|}
\hline \multirow[b]{2}{*}{ Process } & \multicolumn{2}{|c|}{1} & \multicolumn{2}{|c|}{2} & \multicolumn{2}{|c|}{3} \\
\hline & $\begin{array}{l}\text { pre- } \\
\text { test }\end{array}$ & $\begin{array}{l}\text { post- } \\
\text { test }\end{array}$ & $\begin{array}{l}\text { pre- } \\
\text { test }\end{array}$ & $\begin{array}{l}\text { post- } \\
\text { test }\end{array}$ & $\begin{array}{l}\text { pre- } \\
\text { test }\end{array}$ & $\begin{array}{l}\text { post } \\
\text { test }\end{array}$ \\
\hline Execution & 26 & 33 & 30 & 47 & 34 & 33 \\
\hline Orientation & 5 & 5 & 4 & 6 & 9 & 6 \\
\hline Monitoring & 7 & 10 & 16 & 22 & 19 & 13 \\
\hline Regulation & - & 7 & 10 & 13 & 11 & 5 \\
\hline Testing & 1 & 1 & 2 & 6 & 1 & 3 \\
\hline Diagnosing & - & 1 & - & - & 1 & - \\
\hline Evaluation & - & - & - & 1 & - & - \\
\hline Distracted & 3 & - & - & 2 & - & - \\
\hline
\end{tabular}

\begin{tabular}{lrrrrrr}
\multirow{2}{*}{\begin{tabular}{l} 
Subject $N$ \\
\cline { 2 - 6 } Process
\end{tabular}} & \multicolumn{2}{c}{4} & \multicolumn{2}{c}{5} & \multicolumn{2}{c}{6} \\
\cline { 2 - 7 } & $\begin{array}{l}\text { pre- } \\
\text { test }\end{array}$ & $\begin{array}{l}\text { post- } \\
\text { test }\end{array}$ & $\begin{array}{l}\text { pre- } \\
\text { test }\end{array}$ & $\begin{array}{l}\text { post- } \\
\text { test }\end{array}$ & $\begin{array}{r}\text { pre- } \\
\text { test }\end{array}$ & $\begin{array}{r}\text { post } \\
\text { test }\end{array}$ \\
\cline { 2 - 7 } Execution & 24 & 39 & 33 & 30 & 12 & 37 \\
Orientation & 1 & - & 4 & 5 & 4 & 8 \\
Monitoring & 5 & 4 & 23 & 20 & 9 & 11 \\
Regulation & 15 & 15 & 20 & 17 & 5 & 21 \\
Testing & - & - & - & 2 & - & 1 \\
Diagnosing & - & - & 1 & - & - & - \\
Evaluation & - & - & - & 2 & - & - \\
Distracted & - & - & - & 6 & - & -
\end{tabular}


text comprehension. There were no increases in the number of positive selfstatements.

Table 8

Classification of Word Problem Protocols According to Mind and Activity Orientation

\begin{tabular}{|c|c|c|}
\hline Orientation & pre-test & post-test \\
\hline Mind Orientation & 16 & 7 \\
\hline Goal fixation & 11 & 3 \\
\hline Failure fixation & 3 & 3 \\
\hline Planning fixation & 2 & 1 \\
\hline Activity orientation & 2 & 8 \\
\hline Unclassifiable & & 3 \\
\hline
\end{tabular}

\section{GENERAL DISCUSSION}

The two studies reported revealed some differences in regulation processes between performing subjects, who have concentration problems. In study 1 , differences were found between performing subjects in the number of executions and in various "control" processes. Moreover, significant correlations appeared between the process measures and standardized test scores. The differences in mind orientation and distractibility we had expected, however, were too small to be statistically significant in the first study. The scores for mind orientation and distractibility were rather low. In the second study we therefore decided to use a different way of analyzing the protocols with reference to the mind orientation. This approach succeeded.

The training failed to be effective in the first study. No effects of training on performance or process data were found. This may have been caused by the nature of the students, coming from special education. In the second study we intensified and changed the training procedure. We cannot, however, conclude that the difference between pretest and post-test we obtainedin this study has been caused by the training, since there was no control group. As in our previous work (see the introduction section), we did not confine ourselves to product measures. In our view, process training should lead to changes in processing. The changes we found in the process measures and the patterns of mind orientation therefore give some confidence that the training was successful. Thus it seems that training in self-regulation and concentration may be successful, also with students having severe concentration problems. Transfer to the reading comprehension task, however, failed to appear, indicating that explicit transfer instructions should be built into the training program. 
The process measures for the different tasks correlated with each other, indicating that there is at least some individual stability in processes. Although it is reasonable to expect that regulation processes differ for different tasks and task characteristics (difficulty for instance), it is reassuring to note that there is some consistency as well. The correlations between the process measures and standardized test scores can be interpreted as evidence for the validity of the process scores. Specifically, the correlations between mind orientations and impulsivity, fear of failure and achievement motivation, between distraction scores and the standardized concentration test between control and fear of failure and between execution and fear off\&lure add to the validity of the measures. Difficult to interpret, however, is the correlation between intelligence and mind orientation.

The process data on the fractions and problem-solving task in study 1 seem to suffer from the cause and effect problem discussed in the introduction. The fractions posed such great problems for some of the subjects that they tried over and over again, noticing negative interim-results and being rather mindoriented. The problem-solvingtask on the other hand was so difficult for some subjects that they did not do anything at all: processing stopped with hardly any verbalization. It seems, then, that differences in processes depend at least partly on the (subjective) difficulty of the task.

We did not succeed in finding a suitable operationalization for distractibility. Students who were selected because of their concentration problems verbalized only a few task-irrelevant cognitions. This might be an artifact of the thinking-aloud procedure. Probably, the necessity to verbalize keeps students concentrated. In spite of this, a significant correlation with the standardized concentration test was found.

Finally, it should be noted that we do not know which elements of the training were responsible for the obtained effects. Further research is needed to clear this up. In our present studies thinking-aloud protocols are collected with larger samples, using training programs of longer duration.

\section{REFERENCES}

Boekaerts, M. (1982). Onderwijsleerprocessen organiseren: hoe doe je dat? [How to organize learning and instruction processes]. Nijmegen: Dekker en Van der Vegt.

Brown, A.L. (1980). Metacognitive development and reading, In: R.J. Spiro, B. C. Bruce\&W. F. Brewer(Eds). Theoretical issues in reading comprehension. pp. 453-479. Hillsdale: Erlbaum.

Gagne, R.M. (1977). The condition of learning. New York: Hold, Rinehart and Winston.

Galperin, P. Y. (1969). Stages in the development of mental acts. In. M. Cole, \& I. Mailtzman (Eds.). A handbook of contemporary Sovjet Psychology. New York: Basic Books. 
Hettema, P.J. (1979). Psychology and adaptation. Amsterdam: North-Holland.

Klauer, KJ. (1985). Framework for a theory ofteaching. Teaching and Teacher Education, 1, 5-17.

Kuhl, J. (1983). Motivation, Konflikt und Handlungskontrolle. Berlin: Springer.

Larsson, S. (1983). Paradoxes in teaching. Instructional Science, 12, 355-365.

Lawson, M.J. (1984). Being executive about metacognition. In: J.R. Kirby (Ed.). Cognitive strategies and educational performance. pp. 89-109. New York: Academic Press.

Lodewijks, J.G.L.C. (1981). Leerstofsequenties: van conceptueel netwerk naar cognitieve structuur. [Subject-matter-sequences:fromconceptualnetwork to cognitive structure.] Lisse: Swetz \& Zeitlinger.

Olshavsky, J.E. (1976). Reading as problem solving. Reading Research Quarterly, 12, 654-674.

Palincsar, A.S., \& Brown, A. L. (1984). Reciprocal teaching of comprehensionfostering an comprehension-monitoring activities. Cognition and Instruction, 1, 117-175.

Simons, P.R.J., \& Lodewijks, J.G.L.C. (1987). Regulatory cognitions during learning from text. In E. De Corte, H. Lodewijks, R. Parmentier, and P. Span (Eds.). Learning and instruction: European research in an international context, Volume I. pp 357-368. Oxford: Pergamon

Van der Sanden, J.M.M. (1986). Het leren van technische uaardigheden IIndividual differences in learning technical skills]. Den Haag: SVO.

Zak, A.Z. (1980). Een experimenteel onderzoek naar reflectie bij jongere basisschoolleerlingen [An experimental study on reflection in younger pupils of elementary school.1 Pedagogische Studien, 57, 358-365.

\section{AUTHOR}

P. R. J. Simons is professor in the Department of Psychology, Tilburg University, The Netherlands. 
APPENDIX 1

Definition and Examples of Main Categories of Processing Activities
CATEGORY
DEFINITION
EXAMPLES

1. Execution

2. Monitoring

all cognitive and overt activities transforming states of knowledge or understanding in the direction of aimed states

perceiving, interpreting noticing characteristics of executed actions

3. Regulating

\subsection{Planning}

3.2. On-line regulation

4. Orienting

5. Testing

5.1. Testing on understanding

5.2. Testing on knowledge choosing activities and objects on which activities should be performed

regulation on a macrolevel before text processing

regulation during text processing

preparing oneself for the task by inspecting the learning situation, possible activities, goals and own characteristics

all activities leading to information about results of learning

all activities leading to information on understanding

all activities leading to information on knowledge
- reading

- I don't think this is an experiment

-oh yes, I understand -this is very difficult

- if I read this very thoroughly I shall understand it

- l'll just read on, perhaps I shall understand it later

-will there be a test?

- oh, l'm very good at muitiple choice tests

- yes, this seems to follow from this table

- paraphrasing

- reproduction of fragments without reading 
48 CJEC WNTER 1989

APPENDIX 1 (cont'd.)

$\begin{array}{lll}\text { CATEGORY DEFINITION EXAMPLES } & \end{array}$

6. $\quad$ Diagnosing

looking back at a pre- - I just don't understand ceding learning process how this figure has been in order to discover why constructed, but that is resuitsare (not) reached because I am no good at mathematics.

7. Evaluating

judging the total learning process in relation to the goals

- no, I don't understand all of it, but enough to pass the test 


\title{
Profile
}

\section{Applications and Implications of Distance Education: Manitoba}

\author{
Bernard Simand
}

\begin{abstract}
Education in Canada is profoundly influenced by two of Canada's most distinctive features: its vast geography and its small. widely dispersed population. In considering the applications and implications of distance education in Manitoba, it is important to remember that the majority of its population is based in the southem cities of Winnipeg and Brandon. in fact. Manitoba's three universities are located in these two cltles and in addition. two of the three community collegesare also based here. The third, Keewatin community College. is situated in The Pas ond has a satellite campus in Thompson. This demographic condition has provided encouragement for Monitobans to develop and use communications technologies in many diverse activities which will be outlined in this artlcle. In looking at the applications and implications of distance education, it is useful to note that the one common concem underlying all distance education projects in Manitoba is to extend access to educational opportunity. particularly to remote and northem regions.
\end{abstract}

\section{THE HISTORY AND DEVELOPMENT OF DISTANCE EDUCATION IN MANITOBA}

\section{Radio and Television}

One of the earliest uses of educational radio originated in Western Canada in 1941 and is known as "Farm Radio Forum". It served as a radio discussion program and was subsequently adopted in a number of developing countries. "The lessons which were learned from this model, such as the use of forums, multi-media, printed materials, two-way communication and various produetion techniques (drama, interview, panel discussion) were then introduced in India early in 1956, and in Ghana in 1964, with the initiative and sponsorship of UNESCO” (Nwaerondu \& Thompson, 1987). The Canadian experience with educational radio has been quite useful to developing countries in providing experience and general lessons that give support to the view that radio is an effective medium of instruction especially because of its widespread availability in developing countries. 
In Manitoba, the widespread availability of television and video recorders as well as a good cable system has prompted the Department of Education, through the Correspondence Branch, to initiate a pilot project (established in 1984) called Manitoba Educational Television (METV). Their mandate is to enhance in-school and at-home learning opportunities for Manitobans by offering college credit courses, pre-school, primary, junior and secondary school programmes, as well as native education, French language and adult literacy programmes.

METV has partial TV production facilities located in Winnipeg but for the most part has purchased existing programmes from agencies outside the province. Learning support for the in-school programmes is largely the responsibility of the individual schools and teachers, who receive broadcast schedules from METV. It is important to note that students register for courses and receive supportfrom the Department's correspondence branch. Support for the college credit courses is the responsibility of the participating colleges and students register directly with the colleges who provide study support materials, assignments, and tests. METV estimates that their programmes are available to 950,000 viewers through broadcast and cable services. METV continues to grow and expand and serves as a useful means of providing educational opportunities at all levels to Manitobans throughout most of the province.

\section{Telephone (Teleconferincing)}

Teleconferencing is a popular means of delivering educational services to remote sites in Manitoba. One of the pioneers of this communication technology is the University of Manitoba which uses dial-up audioconferencing to link the (teleconferencing) studios at the university with conferencing facilities in 16 communities in rural and northern Manitoba. The U. ofM. reports that the audioconferencing system is in use for approximately 3 hours per day, 5 days per week during the academic year.

Inter-Universities North (I.U.N.), a consortium of the 3 Manitoba universities whose mandate is to deliver university credit courses in the northern part of the province, currently uses teleconferencing for about $40 \%$ of all its course offerings. Both organizations encourage at least one site visit per term by instructors and support their courses by print materials, and videotapes where possible. In addition, each site has a part-time coordinator who supervises the equipment, sets up the conferences and providesbasieadministrative support. Class sizes range from a minimum of 5 students per site to a maximum of 15 .

Another example of the use of teleconferencing in Manitoba is the delivery of teacher education courses through the B.U.N.T.E.P.(Brandon University Northern Teacher Education Programme) to remote northern communities. In January, 1985, the course Survey of Exceptional Children was offered simultaneously in several B.U.N.T.E.P. centres. This project was initiated by PACE. (Program for adult and continuing education) division with the 
assistance of Manitoba telephone system. A network was established to make telephone bridging services and classroom telephone speaker systems available to any programme using teleconferencing to teach adults. Funding was made available to all post-secondary institutions to begin developing courses for delivery by teleconferencing and as a result 32 courses or subjects have been developed. One of the more interesting examples of this development is the joint delivery (University of Winnipeg and Red River Community College) of a course entitled, Human Anatomy and Physiology. The course contains a full lab component that can be completed at home using kits that are mailed to the students. The course is of particular benefit to remote nursing students and qualifies for both university and college credits.

There are also some good examples of the use of distance education at the secondary school level. The Souris Valley School Division has piloted Calculus 305 using a combination of teleconferencing and computer conferencing to its schools at Wawanesa, Hartney and Souris. Students receive and submit assignments through the Manitoba Information Network (MINET) while instruction and interaction is facilitated by teleconferencing. Print materials are supplied by the Correspondence Branch and line charges are paid by the Distance Education and Technology Branch. The schools participating in the project have agreed to give credit to their students who pass the course, and where a student is not affiliated with a particular school, the correspondence division will issue the credit. Lloyd and Karen Paulson, the husband and wife team who wrote and teach the course, estimate a 50-80\% completion rate. An informal survey has indicated that last year's Calculus 305 students are doing very well in university studies.

This pilot project has been so successful that there are plans to expand the number of courses offered through MINET to include 2 courses in Computer Science (BASIC and PASCAL) and a course in grade 12 Physics. One result of the pilot project is the discovery that the following elements are needed to develop a first rate distance education program:

1) appropriately designed courseware;

2) elements that humanize the learning process;

3) tutorial support; and

4) effective evaluation and student tracking.

\section{Computer Conferencing}

Education Minister Roland Penner has announced a 250,000 dollar grant to the Manitoba Computer Assisted Learning Consortium (MCALC) to develop courseware to meet the needs of the province's small schools, and remote northern communities. According to Education Manitoba, in the past year and a half MCALC has marketed 60 new programs in English and French. The Manitoba Information Network (MINET) enables schools to access a host computer by using the telephone system and a microcomputer. It is a joint venture (industry and government) whereby Cybershare provides the re- 
sources of the host mainframe computer, Manitoba Telephone System supplies the telephone and DataPac facilities, and Educational Technology Program (ETP) provides the products and services. The ETP also provides professional development open to all education personnel throughout the province.

In 1983, two professors at University of Manitoba (Drs. Kinsner and Pear) became involved in an on-going project to adapt the principles of Keller's approach to teaching at the post-secondary level known as personalized system of instruction (PSI) to computerization. Their approach, is called computer-assisted personalized system of instruction (CAPSI). Briefly, the main function of the computer is to give tests to students who request them, to assign markers, and to keep track of the progress of each student through the course. CAPS1 has been used successfully at the University of Manitoba for both on-campus and off-campus learning in the area of psychology. It is also being considered for teaching in engineering.

The experience with CAPS1 is important for several reasons. Primarily it suggests that it is a powerful teaching method with wide generality CAPS1 makes it possible to thoroughly monitor, analyze, and evaluate a significant portion of the behaviour and learning in the course. It also opens the door to the next stage of computer-aided instruction, in which the computer will become more intimately involved in the educational process by aiding in the development of course materials and in the evaluation of the student's learning.

In concluding this brief look at the applications and implications of distance education in Manitoba, I have discussed only those projects (with the exception of "Farm Radio Forum") with which I have been personally involved, either directly or by association. In each case, the technology employed, whether computers, television, or teleconferencing, has proven itself to be a useful tool in providing educational opportunities which otherwise would not be available.

A serious implication for all of these projects, and a general concern, is that they have failed to become integrated into mainstream programming. 'Teleconferencing, perhaps the most widespread means of delivering education at a distance, has not been well received in all communities, particularly those that are Northern and Native. CAPS1 has attracted only a handful of students even though regional media, such as C.B.C. North Country radio, has predicted a bright future for computer-assisted learning in the North. As a rule, the public school system has been slow to utilize any of the new communication technologies although nearly every school in the province is linked to a sophisticated computer network (MINET).

One reason for the slow, but steady, development of distance education in Manitoba is the paucity of funding for new and existing programs, which is always a problem in a poor province. As well, in every instance where technology was introduced into the school environment it has not been well received when it has failed to take into account the prevailing social structure (ie. "the way things are done around here"). The I.U.N. experience with 
teleconferencing in the Island Lake region, is a case in point. On the positive side, the Manitoba experience represents an excellent model of cooperation and collaboration between industry and government. Together they have promoted communication technologies as a means of expanding educational opportunity to selected students through distance education.

\section{REFERENCE}

Nwaerondu, N.G., \& Thompson, G. (1987). The use of educational radio in developing countries: Lessons from the past. Journal of Distance Education, 2 (2), 43-54.

\section{AUTHOR}

Bernard Simand is the Secondary School Liaison Officer of Contact North, Thunder Bay, Ontario. 


\title{
The Educational Media Producers and Distributors Association of Canada
}

\section{salutes}

\section{The National Film Board}

\author{
on the occasion of its
}

\section{0th Anniversary \\ 1939 - 1989}

\section{Member Companies:}

Advance Films Ltd. McIntyre Educational Media Ltd.

Britannica Learning Materials McNabb \& Connolly

Mobius Productions Limited

$\begin{array}{ll}\text { CBC Enterprises } & \text { Multimedia Group of Canada } \\ \text { National Film Board of Canada }\end{array}$

$\begin{aligned} \text { Canadian Learning Company Inc. Nationar Googhic Society } & \end{aligned}$

CTV Television Network Ltd. Omega Films Limited

Goldi Productions See Hear Now! Media

International Tele-film Enterprises Ltd. Thomas Howe Associates Ltd.

Kinetic Film Enterprises Ltd. TVOntario

Magic Lantern Film Distributors Ltd. Visual Education Centre

Marlin Motion Pictures Ltd. 
Profile

\title{
Televised Instruction for Post-Secondary Education in Quebec
}

\author{
Gilles Camier \\ Mark Schofield
}

\begin{abstract}
Teaching at a distance in the Province of Quebec is a project shared by various establishments: govemment agencies, community colleges and universities Many of these collaborated to create a consortium in order to distribute their televised courses. C.A.N.A.L. has been offering services to its members since 1984 and today represents a unique case of a private corporation financed exclusively by its membership in the world of distance education establishments. The results of its presence on cable distribution can now be assessed with some confidence. Distance education through television appears to be viable if a technologists approach is favored more than if a broadcaster's dream is pursued.
\end{abstract}

Adult learners in the Province of Quebec have access to televised instruction at all levels through the distribution services provided by CANAL (Corporation pourl'avancement de nouvelles applications des langages/Corporation for the Advancement of New Applications of Languages). CANAL was established in 1982, as a private registered corporation, by Tele-universite, an affiliate of the Universite du Quebec, as a consortium of colleges, universities and public corporations interested in the use of broadcast television for distance education. Since that time membership has grown steadily to include fourteen institutions.

In this article, a short history of the goals and achievements of the corporation over the past five years will be presented.

\section{Historical Background}

In 1982, Tele-universite initiated a project to distribute televised courses and lectures in some thirty municipalities serviced by the various campuses of the Universite du Quebec, using the Community channels of the local cable television facilities. This service was initiated by Mr. Pierre Patry, the coordinator of Les Editions Mediatiques at Tele-universite. In parallel to this, a closed-circuit video network was set-up between campuses of the Universite du Quebec, to allow interactive participation in live courses and graduate semi- 
nars from the various campuses and even on occasions from students in their homes (Lafrance, 19841.

These early experiments demonstrated both the advantages and the limitations of such a system. It was possible for viewers who subscribed to cable services with channel converters to access some twenty-one hours of educational programming per week on a channel shared with Parliamentary debates and other local community programming. Aschedule was provided to students allowing them to tune in at specific times for their courses in much the same way as they would attend live lectures in the classroom. The highlights of this early period included broadcasts of a French version of the Ascent of Man, a course on Publicity, (in which the present mayor of Montreal was a guest speaker), one on Human Biology and several other general courses in the Arts and Sciences. These were also broadcast over the networks of Tele-Metropole and Radio-Quebec.

However, it was soon discovered that only about thirty percent of the households owned the cable converters necessary to allow them access to the designated channels and that the cable companies and broadcasters tended to schedule educational programming in early mornings or late evenings. Students found this very inconvenient and did not respond with much enthusiasm. Also, the production, distribution and shipping of large numbers of duplicate copies of video cassettes, and the problem of having to continually re-negotiate agreements with individual cable companies and broadcasters was a poor longterm foundation for the operation of a distance education service.

These early experiments did, however, help to establish the working principles for a multi-member consortium, and demonstrate the technical feasibility of the organization that was later to become CANAL.

It was recognized by the developers of the system that, to maximize learning, televised instruction should ideally be interactive, and to achieve this video conferencing would be necessary. However, it was also realized that this was beyond the current technical limitations of the system and that televised instruction could only be the first step towards a variety of other approaches involving radio, telephone conferencing, computer conferencing, and telematics, all of which would contribute towards the simultaneous exchange of information and feedback.

From 1982 to 1984, Tele-universite supported the growth of the video distribution service by hiring a coordinator and two employees to take charge of the programme schedule, maintain good relations with the cable companies and broadcasters and organize the shipping and receiving of video cassettes. In spite of the risk of direct competition, other educational institutions outside the Universite du Quebec (1984) were invited to join the organization and pool their production capacities in return for access to the network.

Universite de Montreal (November 1982), Universite de Sherbrooke (February 1983), Universite Lava1 (June 1983) and CEGEP Montmorency (1983) officially joined the fledgling consortium and gave their support to the official application for Letters Patent from the Government of Quebec. They also 
agreed to share the financial burden and make contributions to an independent budget for the new corporation. The coordinator and support staffretained their offices in the Tele-universite building in Montreal but were transferred to the new corporation.

In 198485, the corporation submitted applications for licenses to the Canadian Radio-television and Telecommunications Commission and was granted the following:

1) a license to operate an experimental broadcast television station on channel 62, later to be upgraded (1985-86) to a medium powered transmitter on channel 29;

2) a license to distribute programming to a network of distributors using satellite communications; and

3) a license to enlist sponsors and be permitted to mention their support during programmes.

These three licenses established the legal existence of an educational television network dedicated to formal education, as a supplement to the services already offered by Radio-Quebec.

In 198586, three new members, including Hydro-Quebec, Laurentian University of Ontario, and the Direction des cours par correspondence, (the agency responsible for offering correspondence courses in the Quebec Ministry of Education), joined the consortium, followed in 1987 by Concordia University.

In September 1987, a satellite distribution system was established that allowed the educational network to be received in approximately four hundred and eighty-seven municipalities across the Province by viewers with cable feeds and converters. By this time, because of the enhanced services being offered by the cable companies, the number of subscribers with converters had grown to approximately 85 percent of the total.

The "Canal de tele-enseignement" had finally become a reality, making telecourses available at the post-secondary level to the majority of the adult population in the Province of Quebec.

\section{The Incentive to Innovate}

The technical advances and development of distribution and communications systems of the past thirty years has made the creation of a new television network in the 1980's a fairly straightforward affair. However, for educational institutions with limited resources and facilities, the production of materials still required much of the pioneering effort of the when television was experimental, black and white and mostly live. With a bleak financial future for education, it took courage and perseverance to convince university administrators that money used for original productions for educational television was wisely spent, and that public response, in the form of enrollment in the formal credit courses, would justify the expense. At that time, the trend was for the established educational television networks to offer high budget, well 
polished programme materials, intended for mass audiences. (1984):

The major goals of the organization were stated in the Letters Patent

1) (To) "facilitate experimentation, research and development of new communications media, especially cable distribution and other related technologies;" and

2) (To) "help its members in preparing a programming schedule of educational content and to ensure the integrity of this schedule."

CANAL had been allowed to grow on the assumption that the greater the number of members producing educational programming, the stronger the argument would be for enlarging the distribution system. The resulting growth had two immediate effects:

First, between 1985 and 1986, the number of programme series broadcast increased from 27 to 50 . For the most part, these consisted of 13 titles each with an average duration of one hour. It thus became possible to fill a 24 hours per day, 7 days per week schedule, by repeating each title approximately three to four times per week.

Secondly, the CRTC licenses enabled the corporation to greatly increase its service area. In metropolitan Montreal, the broadcast television transmitter license allowed a greaterproportion of the population to receive the station and also enabled six new cable companies tore-distribute the signal on Channel 23. Channel 23 thus gradually became identified as the "educational television channel."

The natural outcome of this growth was the creation, in late August of 1987, of a provincial educational network consisting of approximately eighty cable companies who agreed to distribute the enlarged 24 hours per day, 7 day per week programme schedule, available via a satellite channel that had been arranged through an agreement between CANAL and the large Montrealbased cable company, Videotron.

The experimental side of the enterprise also developed quite quickly. Production staff became aware of the potential value of events taking place on campus. Conferences, conventions and learned societies provided a wealth of useful material and efforts were therefore made to record proceedings and conduct interviews with guest speakers for later distribution. In 1985, one of these events was broadcast live over the Parliamentary channels across Canada.

A few courses were also broadcast live over the network to allow immediate feedback from students. One of these was organized by Teleuniversite and offered to workers in the construction industry. On Saturday mornings, a wellknown lawyer delivered a series of three hour formal lectures on labour relations to a studio/classroom audience. These were also simultaneously distributed live over the CANAL network. Approximately 1200 viewers participated in the course from their homes and were able to phone in their 
questions and comments during the lectures. Union officials, Government representatives and Employers' Association members were also invited to participate in the studio.

Educational institutions also soon became aware of the potential public relations value of using the CANAL network to present information to the general public on academic programmes, research projects, student services and other activities taking place on campus. This type of programming was useful in maintaining a balance between formal courses, intended for specific audiences, and more general programming aimed at the public at large.

One of the major surprises associated with CANAL's expanded operations was the size of the audiences drawn by many of the formal courses. One introductory course in Astronomy, for instance, which had 250 registered students, attracted an estimated audience of 25,000 viewers between the ages of 15 and 45. The promotional value of this phenomenon was soon realized when the number of applications to the academic department giving the course suddenly increased dramatically.

Over the past five years, televised instruction distribution by the new consortium has grown to become an important factor in distance education in the Province of Quebec. However, although the pioneering days are over, the original goals are only just beginning to be realized to their fullest extent.

\section{Distance Education using Video Communications}

One of the roles of the college and university professor is that of teacher. There is little questioning of the ability to teach in the classroom setting, but there are severe reservations as to whether this skill can be transferred to the television studio. A good teacher is required to present material clearly and systematically, with enthusiasm and in a convincing manner. Personal interaction between student and teacher is also an important aspect that many feel is missingin the distance education mode. It should, however, be borne in mind that there will be a student audience which will interact through assignments, written questions and the telephone. The other characteristics of the good teacher are equally applicable to the telecourse instructor.

It can in fact be argued that, because more careful preparation is required for the televised version of the course particularly for illustrative materials, the effectiveness of the course may be increased.

One of the strongest arguments against the use of low level production comes from the commercial broadcasters who feel that programming of this type adversely affects their reputations. It can, however, be argued further that the incorporation of high level productions, primarily intended for a general audience, into an academic course can be more distracting than useful if not accompanied by very carefully structured support materials.

Many academics feel apprehensive about participating in the presentation of their course in a distance mode on the grounds that the medium imposes limitations on academic freedom and that they feel more open to criticism. On the other hand, many who have given courses at a distance have found it to be 
a very positive experience. The response from students and subsequent contact with students is generally more frequent. Although the risk of criticism is certainly greater, the opportunitiesformakingpublicstatementsonimportant issues are also increased. Academics teaching via television have found that they have become celebrities and are frequently stopped in the street or receive telephone calls from viewers. Many have also found that the experience has become a springboard for career development in the field of post-secondary education.

One factor that has become apparent to producers of educational television is the need to market distance education courses. Even a course given by a teaching expert in the field is not guaranteed an audience. Universities and colleges have learned to advertise their products well in advance through televised promotion, daily newspapers and specialized publications targeted to specific segments of the population.

Various strategies have been adopted to increase the demand for particular courses. Courses have been offered in short intense sessions of six hours per day over a three to four week period. Also, courses have been withheld for a year after the initial offering, in the hope that demand will increase. Another approach has been through direct marketing, involving the instructor traveling across the province to promote the course in person.

One drawback of using a public distribution medium such as broadcast or cable television is the relatively short useful lifespan of a course. Some consider that the average life of a course is from three to four years before major modifications become necessary. There have been exceptions, however, for example, the French version of Dr. Bronowski's "Ascent of Man" series, which has been used as the basis.of an introductory course in social science offered by Teleuniversite, for the past ten years.

Over the past five years, the major characteristics of the approach to televised instruction adopted by the consortium have included a utilitarian approach to production methods, the marketing of courses as products, and making use of available talent rather than bringing in professional performers. The consortium has chosen to concentrate on the development of courses that can be easily modified, and are only intended to be offered for a limited time period, rather than sinking large amounts of their resources into productions intended for extended use.

\section{Students and the General Public}

From 1986 to 1988, members of the consortium distributed an average of fifty series of thirteen titles per year, consisting of 7000 hours of credit courses and 6000 hours of non-credit programming. These courses covered a number of fields: education, religion, management, pure and applied science, health science, social science and computer science. The costs of production, licensing agreements, contractual arrangements with authors and teachers, collection of student fees and the packaging and marketing of the course materials remained the responsibility of the individual institution.

So far, the response from both students and the general public has been 
very encouraging. From January 1986 to December 1987, over 17,000 students registered in seventy nine courses offered by three institutions (Table 1).

Table 1

Student Enrollment for 1986 and 1987

\begin{tabular}{lccccc} 
& \multicolumn{3}{c}{1986} & \multicolumn{2}{c}{1987} \\
& & & & \\
& & Courses Students & Courses & Students & Total \\
\cline { 2 - 6 } Universite Laval & 6 & 792 & 13 & 1,463 & 2,255 \\
Univ. de Montreal & 12 & 1,240 & 14 & 1,078 & 2,318 \\
Tele-universite & 17 & 5,505 & 17 & 7,145 & 12,650 \\
\cline { 2 - 6 } TOTALS & & 7,537 & & 9,686 & 17,223 \\
\hline
\end{tabular}

The instructional methods used in these courses have been highly varied. Some courses consisted of straightforward sixty minute lectures supplemented by notes; others were essentially print-based courses using the television network for distribution of complementary materials; and others consisted of live lectures, delivered to a small studio audience but allowing interaction from students in their homes, via telephone.

The actual size of the audience, consisting of both students and members of the general public, viewing a particular programme, is very difficult to estimate. As of now, the broadcast survey companies have been unable to produce reliable viewer statistics for the specialized services carried by the cable companies. Apparently, questions such as channel and title of programme watched received too many confusing and contradictory answers to be of much value. The only available data are the Neilson ratings for the programmes from the consortium that are re-broadcast over the Radio-Quebec network. These are summarized in Table 2 (on following page).

Considering that none of these courses had an enrollment of more than three hundred students, the bulk of the audience consisted largely of members of the general public who were apparently interested in the specialized materials being presented.

As a novice broadcaster, the educational network is quite proud of its achievements. The positive public reaction has provided sufficient reason for the network to continue to develop its services and expand its coverage. With this in mind, discussions are being held with universities in other parts of Canada and world, so that expertise and programme materials may be shared and exchanged.

In spite of the fact that it is not yet possible to enrol in courses at a distance 
Table 2

Neilson Ratings for 1987-88 as Compiled by Radio Quebec

$\begin{array}{lr}\text { Programme Title } & \text { Rating } \\ \text { Universite Laval } & 11,000 \\ \text { - Gerer sa foret } & 4,000 \\ \text { - Formation continue en science de la sanee } & 2,000 \\ \text { - La mythologie Greco-Romaine } & 3,000 \\ \text { - L'homme et ses aliments } & 1,000 \\ \text { - Lecture du Nouveau testament } & 7,000 \\ \text { - Sante et securite au travail } & 3,000 \\ \text { - De familles en families } & 6,000 \\ \text { - Psychologie de L'apprentissage } & \\ \text { Universite de Montreal } & 4,000 \\ \text { - Grands courants de l'art: l'heritage occidental } & 1,000 \\ \text { - Communication et organization } & 3,000 \\ \text { - Les prophet d'lsrael } & 7,000 \\ \text { - Andragogie, domaine de pratique sociale } & \\ \text { Ecole nationale d'administration publique } & \\ \text { - L'ENAP presente } & 1,000 \\ \text { University du Quebec a Montreal } & \\ \cdot \text { La gestion au feminin en France } & 1,000 \\ \text { Tele-universite } & \\ \text { - Le corps humain } & 3,000 \\ \text { - Evolution de l'homrne } & 1,000 \\ \text { - Science et cultures } & \end{array}$

leading to a full certificate, student enrollment has been quite respectable. However, so far, the venture has not generated very much in the way of revenues. The students' fees and grants only cover the costs of production and support services.

\section{Conclusion}

In just five years, the small auxiliary videotape distribution service of Tele-universite has evolved into a consortium of educational institutions, producingmaterialsfor distance education, providing liaison with the national broadcast networks, and assisting in the recruiting of thousands of new students.

Distance education through television is now part of Quebec life. From session to session, there have been marked improvements in programme 
quality and a gradual diversification of instructional approaches. (Broadcast coverage which was initially restricted to the larger cities, is now available in all communities in Eastern Canada via satellite). The technology is no longer the limiting factor to the availability of systems for the distribution of distance education to the adult population.

The original goal of using interactive communication for distance education has not been achieved and still requires further development. However, experiments currently under way are intended to demonstrate the means by which this objective may be realized in the near future.

\section{REFERENCES}

LaFrance, A. (1984). La t\&vision educative. L'interconnexion entre les systemes d'education et de communication a un reseau. Montreal: $L e$ Devoir, 6 juin.

Letters Patent, (1984, July 9), Libro C-1166, p.3.

Universite du Quebec (1984, octobre). Le reseau de television educative. Quebec: Vice-presidence a la planification, Universite du Quebec.

\section{AUTHORS}

Gilles Carrier is currently Director General of Canal de tele-enseignement in Montreal and a Ph.D. student in Educational Technology at Concordia University.

Mark Schofield is Director of the Audiovisual Department in Concordia University. 


\section{CALL FOR PAPERS}

\section{The Canadian Journal of Educational Communication}

invites papers in the area of:

\section{Interactive Learning Technologies}

to be published in a special issue devoted to this topic in the Fall of 1989. Topics within this area may include, but are not limited to: computer-aided learning; interactive video; intelligent tutoring systems; hyper-media (e.g., HyperCard); expert advisory systems; telecommunications; computer conferencing; and smart systems.

Papers may take the form of reviews of literature, descriptions of approaches or procedures, descriptions of new applications, theoretical discussions or reports of research. For details on the preparation and submission of manuscripts, please refer to Information for Authors on the inside back cover of this or any issue of CJEC. Except in unusual cases, manuscript length should not exceed 20 pages. All manuscripts received by the editor will be reviewed by a panel of judges.

Please address all enquiries or manuscripts to:

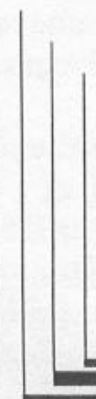

Dr. Richard A. Schwier

CJEC Guest Editor

College of Education

University of Saskatchewan

Saskatoon, SK S7N 0W0

(306) 966-7641 


\title{
Research Technique The Slope Test: Applications in Formative Evaluation
}

\author{
Jon Baggaley \\ Aaron-Henry Brauer
}

\begin{abstract}
Logistical problems associated with formative evaluation reduce the reliability of pre-test/post-test comparisons as a basis for understanding educational effects, A paradigm shift is recommended. focussing attention upon the adjusted post-test scores which are produced when the 'slope test' for homage neity of regression is conducted In a between-groups analysis of covariance.
\end{abstract}

\section{PROBLEMS IN FORMATIVE EVALUATION}

Formative evaluation is often a rough-and-ready process, in which the rigour associated with conventional research methods is unattainable. The essence offormative evaluation is to assess the impact of educational materials while there is still time for the production team to make modifications. The process must therefore be as speedy as possible. It may also have to be organized amid extreme pressures, especially in complex contexts such as TV production where a study can be required with little or no notice.

In this situation, a major problem is that of audience sampling. If the intended audience of the educational product is specific, the impact of the materials upon a representative audience sample can be assessed with relative ease. A simple post-test can establish the sample's overall reactions to the material; and the added use of apre-test can verify that specific knowledge was imparted by the educational materials (or impeded), or that attitudes/behaviour were changed (Campbell \& Stanley, 1966). Pre-test scores in their own right can indicate that the audience was in possession ofcertain facts, attitudes or behavioural traits before the material was presented, and that aspects of the latter are therefore redundant.

As the target audience becomes more diverse, however - comprising both sexes, different age and educational levels, and wide-ranging attitudes or aptitudes - the effects of educational treatments upon it become harder to discern. At the pre-or post-test levels individually, even simple effects may go unnoticed, owing to sampling biases beyond the evaluator's control. The

CJEC. VOL 18, NO. 1, PAGES $65 \cdot 68$, ISSN $0710 \cdot 4340$ 
problem is compounded when pre- and post-test scores are compared, since pre-to-post shifts may well be concealed by prior differences between separate audience subgroups.

In many evaluation studies, of course, it is possible to anticipate the critical audience variables on which treatment effects will depend. Audience subgroups can then be identified, and the pre- and post-test scores of each (e.g., men versus women) compared. Treatment effects on subjects with good reading ability, for example, may be compared with those observed on poor readers, and so on. The resulting evaluation scheme is the "pre-test/post-test multiple-group" design (PPMG), applied in aptitude-treatment interaction studies (Salomon, 1979).

\section{APTITUDE VERSUS TREATMENT EFFECTS}

In a PPMG context, the confounding effects of extraneous audience aptitudes can be identified via a statistical procedure known as the "slope test" (Cronbach \& Snow, 1977). The test is particularly applicable in formative evaluation studies owing to the common logistical problems encountered in this area. It is necessary for the slope test manoeuvre that the pre- and posttest measures be precisely matched, with respect to content validity as well as to the individual subjects in the sample.

When, within a particular audience group, a significant treatment effect is present, the pre- and post-test scores of the group will be relatively uncorrelated, and the "slope" of the scores, plotted graphically, will be flat. If the treatment effect is dominated by audience aptitudes, however, the pre- and post-test scores of the group will be highly correlated, and the slope will be steep. If different treatment effects occur in separate audience subgroups, the slope test will indicate that the groups' pre/post slopes are significantly different, and that the treatment effect cannot be explored in terms of pre-topost response shifts, owing to violation of the statistical assumption of homogeneity of regression.

In the PPMG context, the slope test thus provides the same safeguard against an unreliable treatment effect as the interaction term provides in oneway analysis of variance (Tabachnik \& Fidell, 1983). If the result of the slope test is not significant, the evaluator may proceed to examine the educational treatment effect via an analysis of covariance (ANCOVA). With the pre-test scores as covariates, and audience differences as the independent variable, the sample's post-test scores are adjusted to take account of between-subgroups prior differences. Differences between the adjusted post-test scores of the subgroups can then be directlyattributedto the treatment. (N.B. The slope test and subsequent one-way ANCOVA are available within a single procedure when PPMG data are analyzed via the BMPDIV statistical package. 


\section{An Example}

The utility of the slope test and covariance procedure was noted in a recent evaluation study of an educational film about AIDS (Baggaley, Glegg \& Brauer, 1989). Figure 1 indicates the confounding observed in the study between the treatment effect (pre-test to post), and the attitude differences of separate audience subgroups (high-risk subjects vs. low-risk). The attitude measure in question was one of tolerance towards the rights of persons with AIDS in society. The slope test indicated that the pre/post effect of the film on this measure was nsignificantly different in the two AIDS-risk groups.

Figure 1. Adjustment of Post-test Scores after Non-significant Slope Test.

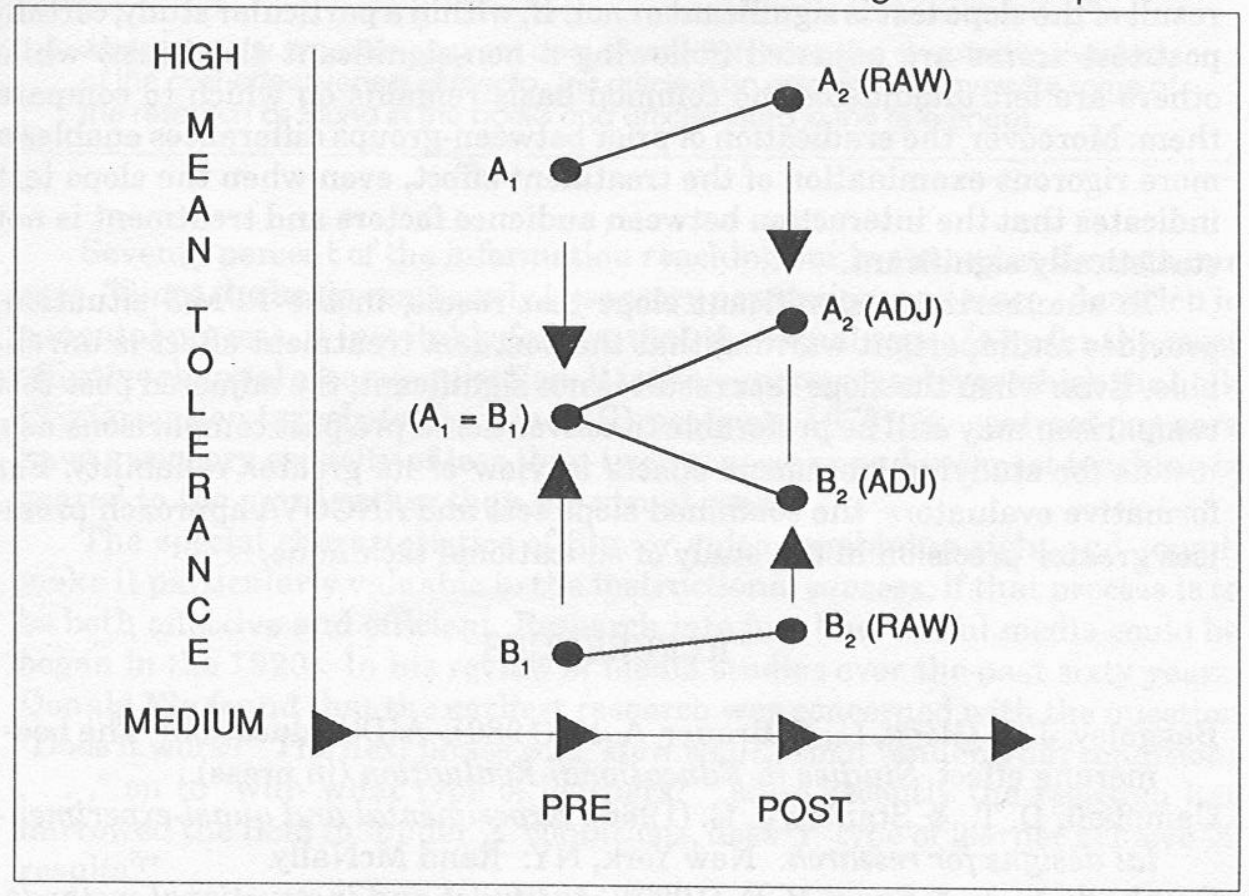

As the pre-test mean scores show, high-risk subjects (A) were markedly more tolerant than low-risk subjects (B). At the post-test level (raw means), this effect seems to have increased. However, the precise effect of the educational treatment on the post-test scores is not yet apparent. In order to isolate it, the between-groups pre-test difference was reduced via one-way ANCOVA to zero $\left(A_{1}=B_{1}\right)$, and the post-test mean scores $\left(A_{2}\right.$ raw and $B_{2}$ raw) were adjusted accordingly. The treatment effect can now be inferred from the difference between the adjusted post-test means $\left(A_{2}\right.$ adj $-B_{2}$ adj); in this instance the difference was found to be statistically significant. On this basis, Baggaley, Glegg \& Brauer concluded that the educational film had polarized the attitudes of high and low-risk viewers with respect to social tolerance, and had thus done more educational damage than good. 


\section{APARADIGM SHIFT}

The covariance analysis of pre/post-test data involves a style of questioning to which formative evaluators are unaccustomed. Instead of asking the usual question ("Are the pre and post-test responses different?"), the analysis asks "Are the post-test responses of audience subgroups different, all pretest factors being equal? "The "paradigm shift" from the first question to the second anticipates the statistical problems with which formative evaluation studies are plagued.

The identification of treatment effects via ANCOVA-adjusted post-test scores may be desirable in some formative evaluation situations whether the result of the slope test is significant or not. If, within a particular study, certain post-test scores are adjusted (following a non-significant slope test) while others are left unadjusted, no common basis remains on which to compare them. Moreover, the eradication of prior between-groups differences enables a more rigorous examination of the treatment effect, even when the slope test indicates that the interaction between audience factors and treatment is not statistically significant.

To summarize, a significant slope test result, in the PPMG situation, provides an important warning that the post-test treatment effect is unreliable. Even when the slope test result is not significant, the adjusted post-test comparison may still be preferable to conventional pre/post comparisons as a means for studying treatment effects in view of its greater reliability. For formative evaluators, the combined slope test and ANCOVA approach promises greater precision in the study of educational technique.

\section{REFERENCES}

Baggaley, J. P. Glegg, L., \& Brauer, A. H. (1989). AIDS education: The boomerang effect. Studies in Educational Evaluation (in press),

Campbell, D. T., \& Stanley, J. C. (1966). Experimental and quasi-experimental designs for research. New York, NY: Rand McNally,

Cronbach, L. J., \& Snow, R. E. (1977). Aptitudes and instructional methods. New York: Wiley.

Salomon, G. (1979). Interaction of media, cognition, and learning. San

Francisco, CA: Jossey Bass.

Tabachnik, B. G., \& Fidell, L. S. (1983). Using multivariate statistics. New York, NY: Harper \& Row.

\section{AUTHORS}

Jon Baggaley is a Professor of Education, and Director of the M.A. Programme in Educational Technology at Concordia University, Montreal, Quebec.

Aaron-Henry Brauer is a Master's candidate in Educational Technology in the same Department. 


\title{
Media Manager's Column Letting AV Media Work For You
}

\author{
Jarvis Stoddan
}

Abstract: Over the years. a great deal of research has been devoted to the study of the cost effectiveness of media. This article is on attempt to summarize some of the research as found in the books and articles listed in the references.

Seventy percent of the information reaching our brain comes through our eyes. "Since the brain reacts only to sensory perception, and since education is a mental process, it inevitably follows that the visual sense is by far the most effective channel ofcommunication. It is the 'supereyeway' over which the bulk of information travels to the mind" (Braselman, 1978). By contrast our ears have a sensory capacity of less than twenty percent and yet most teaching is geared to the aural rather than the visual sense.

The special characteristics of film or video, combining sight and sound, make it particularly valuable to the instructional process, if that process is to be both effective and efficient. Research into just how useful media could be began in the 1920s. In his review of media studies over the past sixty years, Donald Elyfound that the earliest research was concerned with the question "Does it work?" The next phase was 'How well?," then "under what conditions ...?" on to "with what type of learners?" More recently the research has narrowed the field to "under ' $\mathrm{X}$ ' conditions, does ' $\mathrm{Y}$ ' type of learner achieve ' $\mathrm{Z}$ ' results?"

After all these years of research, several conclusions seem possible:

\footnotetext{
How the medium is used may be more important than the choice of medium.

Learning seems to be more affected by what is delivered than by the delivery system.

Most research indicates that the way media are used till determine the learning outcomes.

... it is the design of the software that can bring about consistently quality instruction rather than the medium used. (Software means subject matter, content, structure, not medium). (Ely, 1966)
}

CJEC, VOL. 18. NO 1, PAGES $69-73$, ISSN $0710-4340$ 
There has been no research to substantiate one medium being superior to another.

According to Molstad (1974), there is significant evidence to justify the following claims when instructional technology is carefully selected and used:

1) Significantly greater learning often results when media are integrated into the traditional instructional program;

2) Equal amounts of learning are often accomplished in significantly less time using instructional technology;

3) Multimedia instructional programs based upon a "systems approach" frequently facilitate student learning more effectively than traditional instruction; and

4) Multimedia and/or audio tutorial instructional programs are usually preferred by students when compared with traditional instruction.

Braselman adds that most studies have shown that the use of films greatly speeds up training (by 20 to 25\%) without loss of training quality.

'There is a relatively substantial literature base dealing with the cost effectiveness of instructional technology" (Caffarella, 1977). However, according to Wilkinson (1983), 'The effectiveness of media is not founded in any variable that is inherent in the devices, but in how they are used. This implies that technology is a technique of designing instruction, rather than the more common perception of technology as machine."

Wilkinson goes on to describe three patterns of media use. The first one is the additive approach where a film or video is added to regular instruction, but is "not necessary for the achievement of basic instructional outcomes." The result is not cost effective. Unfortunately, according to To Improve Learning: An Evaluation of Instructional Technology (1970), a report to the U.S. President and Congress, "instructional technology is largely supplementary ... (and) generally employed intermittently."

A second approach is the integrated one. Here, carefully selected materials are integrated into regular instruction and provide an essential element in that instruction. In this situation the teacher and the media are interdependent. There is significant increase in student achievement, and the result is cost effective.

Finally, in the independent approach, instruction is redesigned so that basic instructional outcomes are achieved through the active and passive interaction of students and instructional materials without the direct intervention of the teacher. There is a major initia1 cost to the school system, but this approach has the greatest potential for increasing the cost effectiveness of education.

In an article entitled How to Involve Learners in Your Lectures, Guild (1983) suggests eighteen different methods, but topping the listis "visual aids." Shecommentsthatwhile 
deal with values, beliefs or attitudes. Attitudes are changed more significantly through the use of films than by any other method of instruction.

As has been said a number of times before, the way media are used is extremely important. Involvement is needed, both by the teacher and by the students. By delivering an oral introduction the teacher becomes part of the film experience. Use of questions both before and immediately after the screening, as well as showing the material a second time, can increase the amount of information learned.

While it can be seen that carefully selected and properly used media can be cost effective, from time to time barriers arise that need to be overcome. One of these is availability; effective use depends on adequate availability. In addition the media specialist can play an important role in helping teachers. In a 1981 study of twenty high schools in Wayne County, Michigan, it was discovered "that sixty-four percent of media specialists had never been in a classroom when media were used. Forty-one percent of the media specialists said they did not know how teachers actually used AV materials" (Day, 1987)

Studies indicate that teachers' attitudes towards media are influenced by the supervisor/principal, not by the media specialist. In fact the amount of support the teacher receives from the supervisor is an important factor in predisposing the teacher to use media. While all this is true, research also indicates that the media specialist is in the best position to be a catalyst, and act as a force for change. The specialist needs to go out of his/her way to make it as easy as possible for teachers to use media. This may mean getting rid of barriers to easy use, or it may mean going into the classroom to give the teacher a hand. Finally, training in selecting and using media is very important in developing in positive teacher attitudes towards media.

\section{ACCELERATING USE OF MEDIA IN BUSINESS GOVERNMENT AND MEDICINE}

The use of media in these fields started to accelerate in the sixties, and twenty years later it appears to be increasing at the rate of $20 \%$ per year (Thomas, 1980). A percentage breakdown of where media are used by business, government and medical organizations would look something like this:

$\begin{array}{ll}\text { Employee communications } & 10 \% \\ \text { Management communications } & 10 \% \\ \text { Marketing communications } & 10 \% \\ \text { Continuing education and training } & 70 \%\end{array}$

Of these four areas, the fastest growing is management communications.

Why does business spend ever increasing amounts on media when educational spending seems to be in decline? 
Two reasons: first, according to Thomas, economic analysis of communication cost effectiveness is the basis for making media decisions in most organizations. Some companies may spend in excess of two million dollars per year on video communications, hut this expenditure is more than made up in savings on education staff, travel, time spent in meetings, etc. One major company where equipment maintenance was a problem was able to cut costs by 1.6 million dollars through one program where media was an essential component.

Second, these organizations are communicating through AV media, not using film or video as an add-on to the educational process as happens in most school situations. For this reason they are using media in the most cost effective way possible and theeconomic benefits are obvious.

It seems obvious that AV media have the potential to save money for educational institutions just as they have proven to do so for business. But there are conditions, and here are some of the most important ones:

1) AV media must be integrated into the learning process and not just used as an add-on;

2) the teacher is the most important part of the "film experience," giving an oral introduction with questions before and after the showing;

3) media specialists need to take an active role in helping teachers make the best use of media; and

4) supervisor/principal support and encouragement are crucial if the media program is to become cost-effective.

Is it so surprising that audio-visual instruction can be such a powerful medium? These studies confirm what was expressed long ago in this Chinese. proverb:

"I hear and I forget, I see and I remember, I do and I understand."

\section{REFERENCES}

Bowie, M. M. (1983, April). Media utilization in the classroom. Drexel Library Quarterly, 17-19.

Braselman, H. P. (1978, September). Instructional film: Asset or liability. Audiovisual Instruction, 14-15.

Caffarella, E. P. Jr. (1977, August). The cost effectiveness of instructional media technology in higher education. Educational Technology, 17, 22-26.

Day, J., \& Scholl, P. (1987, January). Media attitudes of teachers can be changed. Educational Technology, 27 (1), 23-24.

Ely, D. P. (1986). Educational technology research: A status report on classroom applications. Paper presented in Tokyo.

Flammer, G. H. (1987, February). A Model of learning and learning Engineering Education, 268-273. 
Greenfield, P. (1985, October). Multimedia education: Why print isn't always best. American Educator, 9(4), 596-607.

Guild, P. B. (1983, April). How to involve learners in your lectures. Training, 43-45.

Molstad, J. A. (1974, Winter). Selective review of research studies showing media effectiveness: A primer for media directors. ACVR , 22(4), 387-407.

Nelson, C. E., Prowar, T., \& Tucker, D. (19'78, January). The decline of traditional media and materials in the classroom. Educational Technology, 48-49.

Ontario Ministry of Education (1982). Partners in Action. The Library Resource Centre in the School Curriculum. Toronto: Author.

Schramm, W. (1977). Big media, little media: Tools and technology for instruction. Beverly Hills, CA: Sage Publications.

Sharon, D. (1988). The Renfrew Quality Education Project: Teachers views after the first year. Canadian Journal of Educational Communication, 17 (l), 53-62.

Springer, L. (1981, November). Put media at the core of the curriculum. Instructional Innovator, 24.

Tickson, S.G. (1970-71). To improve learning: An evaluation of instructional technology (Two volumes). New York, NY: Bowker.

Thomas, W. (1980). Using media in business, government and medicine, New Directions for Continuing Education, 5, 55.63.

Wilkinson, G. L. (1980). Media In Instruction: 60 Years of Research. Association for Educational Communications and Technology, Washington, D.C. Report.

Wilkinson, G. L. (1983, September). Using media to make instruction more effective. Instructional Innovator, 17-19.

\section{AUTHOR}

Stoddart is the Executive Director of the Educational Media and Producers and Distributors Association of Canada. 
74 CJEC WNTER 1989 


\section{Book Reviews}

Three monographs on the application of technology in education, published by ERIC Clearinghouse, are reviewed.

Instructional Facilities for the Information Age by Frederick G. Rnirk, Syracuse, NY ERIC Clearinghouse on Information Resources, Syracuse University, 1987.

\section{Reviewed by Gary M. Boyd}

This booklet is intended as a practical aid for anyone who has to design and equip places (laboratories, carrells, classrooms, etc.) for instruction. It should prove invaluable to educational technologists who are involved in instructional project design or management, and also to school architects.

Fred Knirk has pulled together in one place, in succinct form a great deal of research-based information on ergonomics and comfort for various kinds of individual and group learning places especially where computer and audiovisual/video technology is employed.

Although the main reasons for the lack of impact of educational technology have to do with the shortage of good software of all types, and with the ideology of schooling, a very important secondary cause of rejection is discomfort. Moreover much such discomfort is subliminal, involving fatigue and lowered cognitive skills without any obvious cause. The net effect then can be aversion toward the use of the new instructional technology without awareness that the real causes are in improperly designed facilities.

Cold fluorescent lighting is tiring, erratic noise from adjacent users interferes, there is not enough space in carrells for working materials, poor ventilation is fatiguing, etc. Most of these kinds of discomfort are obvious to any thoughtful person, and the remedies are usually fairly obvious. But the remedies require planning, and politicking, and sometimes cost appreciably more than just putting equipment in existing rooms.

This booklet is especially valuable because it brings together evidence that comfort and ergonomic factors do make a real difference to learning which is 
worth paying for. This evidence can be used by a project developer to argue for proper design.

This monograph also supplies a great deal of critical design information about particular factors, where everyday common sense beliefs are often wrong, (e.g., typically, people put VDTs on top of computers so that you have to look up at them, whereas looking down slightly at a 25 degree angle is actually optimal for minimum fatigue).

An interesting aspect of the research is that there are distinct differences in comfort requirements for adults from those for adolescents, as well as the obvious differences for young children, (e.g., air temperature for adults: 70-78 degrees Fahrenheit for children 65-70 degrees Fahrenheit.)

The research on colour is particularly interesting, and relevant now that psychedelic computer graphics are appearing in courseware. In displays, the extremes of red and blue-violet should be avoided for important information and to minimize fatigue. Contrast between equipment and room walls etc., should be minimal. Social areas (arousing hues) should have different colours than secondary-school classrooms or laboratories (blue-green, green, grey or beige). Fluorescent lights if used should be of the full spectrum type, and should be situated and louvered so as not to reflect from VDT screens, to avoid fatigue and eye strain.

The collection of information is quite comprehensive, and is up-to-date to 1986 at least.

After reading the booklet through, one is left with the feeling that virtually every existing instructional facility could be appreciably improved by applying the knowledge given.

Knirk's monograph should be on the working bookshelf of everyone who designs, develops or manages teaching-learning facilities.

REVIEWER

Gary M. Boyd is a professor in the Graduate Programs in Educational Technology at Concordia University, Montreal, Quebec. 
A Reappmiaal of Instructional Television, by M. Cambre, Syracuse, NY ERIC Clearinghouse on Information Resources, Syracuse University.

\section{Reviewed by Jon Baggaley}

The publication of this monograph is timely, for it reminds us that television is still the instructional medium with the widest distribution capability in the developed world. Of late we have been distracted from the benefits of instructional television by the exciting potential of the newer computer-based technologies. However, as the full applications of the computer in education become absorbed, the potential of computer-based TV methods becomes more fully appreciated. Television continues to evolve as the medium which, as McLuhan noted, carries all others.

The rapid evolution of ITV has been only too obvious to readers of recent CJEC issues. Television is seen as a prime carrier of broadcast education, of distance and interactive education, of individualized and group instruction in all their forms. Marjorie Cambre herself is a believer in television's overweening scope. Her previous investigations of interactive video have already anticipated the computer-based TV ways of the future. Her work in formative evaluation has generated immense interest in this key approach to the development of future ITV production methods.

In the current monograph, however, Cambre refrains from imaginative futuristic assessments of instructional television mainly, to avoid appearing "something like another new technology evangelist" (p. 49). Since the nominal purpose of the book is to reappraise instructional television, one is tempted to assume that this decision is deliberate. However, it becomes clear that an assessment of ITV's future scope is at least part of the books purpose; and in this light the failure to discuss a medium as potent as videodisc before its last page is to say the least disappointing.

The book provides a digest of developments in the ITV field, with three emphases: historical (pp. 1-29); research /evaluation (pp. 30-37); andfuturistic (pp. 38-50). (It is in fact a review of other reviews, and this book review thus becomes a 3rd-generation commentary on the topic!) The book's concentration upon ITV's past may in part be due to its sub-title: An Information Analysis Project; for there is obviously less information around to analyze concerning ITV's exciting future possibilities than there is about the mistakes of its past. Nonetheless, the range of information which the book analyzes is to say the least scanty.

The book's strength lies in its coverage of ITV's usage in high school education, and its chosen emphases may well have been motivated by a concern to speak to the school-teaching audience directly However, this is no reason to exclude almost totally a) educational technology journals; and b) non-American literature. A reference to the CJEC journal breaks this pattern on both counts (though in the process the journal is renamed CJEM). The Journal of Educational Television is not quoted at all. Since the book is published by the 
dozen of ITV literature search facilities -ERIC -these omissions are to say the least puzzling.

The book implies that many critics of instructional television (largely left nameless) have described it as "a failed medium" (p. 1); and it quotes perhaps a little too generously such people's views that those involved in ITV research and evaluation are primarily to blame for this. The results of 30 years of ITV research (p. 37) appear to have contributed little beyond a vague awareness that the medium can teach (sometimes), and that good ITV lessons will teach students better than bad TV lessons. There is clearly an immense research literature in Dr. Cambre's own field of instructional design which could have been mentioned at this point. Instead, the book turns to an inspection of ITV's future, mainly through the eyes of the ITV Futures Planning Group, an unpublished think-tank of TV broadcasters and bureaucrats at the Corporation for Public Broadcasting, apparently still in session five years later.

Now, when groups such as this get together, they are almost certain to gloss over the achievements of TV researchers and instructional designers, for they are almost certainly ignorant of them. TV practitioners do not always like these approaches, believing that they threaten their creative license. Numerous such forums have taken place in the past, and Dr. Cambre alludes to "the grandiose goals and impossible promises" which they have traditionally set (p. 39). The conclusions of the ITV Futures Planning Group - setting priorities, encouraging good research, providing a creative atmosphere, etc. etc., - are clearly the same old lip-service and hardly worth reappraising. The fact that they are quoted at all, and in some detail, adds to the books oddity.

The disappointments, scantiness, puzzlements, andgeneral oddness of the book may conceivably be due to the interests of its sponsor, the U.S. Department of Education. Like the bureaucrats of broadcasting, government officials have little interest in radical evaluation methods or proactive fascination for new technologies. By tradition they favour the reactive approach; and the books attempted justification of old ITV styles within the U.S. school system may certainly have been regarded by them as expedient. Caught between the bureaucrats of two industries, the author has possibly found herself bound to discuss ITV according to an old-style definition, and unable to emphasize any of the interesting perspectives which have previously occupied her.

This theory may be all wrong; but only such a theory could explain why an academic with an interesting prior bibliography should have produced such a restricted set of emphases. The book provides an interesting account of ITV's historical uses in the U.S., and in that respect it is a useful reappraisal. For the devotees of instructional television, however, it holds nothing which is new. At the same time, for cynical observers of the medium, it holds little which is likely to convert. It seems to "reappraise Caesar and to bury him" at the same time.

\section{REVIEWER}

Jon Baggaley is a professor in the Graduate Programs in Educational Technology at Concordia University, Montreal, Quebec. 
Educational Technology: The Closing-In or The Opening-Out of Curriculum and Instruction, by Kenneth Komoski, Syracuse, NY: ERIC Clearinghouse on Information Resources, Syracuse University.

\section{Reviewed by Cheryl Amundsen}

Kenneth Komoski, like generations of educational critics in the United States, argues that the inflexible, uniform curriculum of public education does not reflect the needs of either the individual learner or present day society In his view, the emergence of the textbook industry in 1840 provided the major elements for a rigidly structured, product oriented system which still dominates in most schools today. Pointedly, he attacks the utilization of the newer or advanced technologies in the schools, a practice which has been hailed in many corners as being more learner centered and interactive. Komoski contends that educators have missed the potential which the newer technologies offer because they have employed them simply to do more of the same, but more efficiently. He believes that, "curriculum as an adaptive, equilibriumseeking force that keeps the school in touch with the present while moving it into the future, is no longer an option; it is an imperative" (p. 15).

Komoski supports his arguments through the discussion of three dichotomous perspectives: systematic versus systemic curricular perspectives; exploitative versus cooperative instructional technologies; and closed-in versus opened-out curriculum and instruction. He then proposes some directions and solutions which, unlike those proposed by other educational critics, may be significant enough to equal the magnitude of the reforms he recommends.

Komoski describes current thought and practice concerning curricular development as systematic. He argues that instead, it should be systemic. He contends that in most schools: 1) the emphasis is on the ends (the test scores) rather than the means (the learning process); 2) the learning process is generally perceived as working through a procession of commercially produced instructional materials or systems; and 3) the commercially produced materials are based on fixed learning objectives with a narrow focus which excludes a wholistic perspective of the learning process. Komoski would like to see curriculum development become a dynamic, systemic process; a cooperative effort which includes the educators, the learners, the parents and the community.

A primary interest of Komoski's has been the instructional resources and materials which are used by teachers and learners. From this perspective, he is concerned that the newer instructional technologies, which have so much potential for positive impact, are being generally misused. He makes a distinction between technologies which are exploitative in nature and those which are cooperative "with the aesthetic and rational capabilities of human nature" (p. 9). He maintains that the first view of technology has dominated in western society: "it has been the exploitative technologies, with their undeniable and demonstratable efficiency and effectiveness, that have shaped our thinking about, and our practice of all technologies -including those such as 
medicine and teaching that, presumably, function more effectively when practiced as cooperative technologies" (pp. 10-11). He argues that uses of instructional technologies are generally exploitative in nature because the primary mode of utilization is "drill and practice" linked to specific objectives and standardized achievement tests determined by the commercial creators of the technology. Komoski discerns that educators are generally pleased with these efforts because they have been told that productivity should be their major concern and they have defined productivity as doing more of the same faster and better.

Systematically structured curricula which employ instructional materials and technologies in an exploitative manner characterize what Komoski labels "closed-in" curriculum and instruction. He states, "by committing to a systematically packaged, closed-in curriculum program that promises results on a pre-determined measure, it is easy to ignore the need to develop an opened-out systemically-evolving curriculum designed to educate students for an unknown future, rather than to train them to do well on today's known tests" ( $p$. 14).

Komoski believes that to remedy the situation, administrators and teachers must simply become more involved and work in cooperation with the learner and the community in the development of relevant curricula. He recommends that the structure, stated objectives and instructional methods underlying any integrated system, whether textbook or computer-based, should be examined before making a decision to purchase. He strongly believes that commercially produced instructional materials should not comprise the learner's total education, but that different learning experiences should be designed, for example, community-based experiences or peer-teaching experiences. He cites the need "to carefully think through the educational purposes of the school and the need to design, select, and arrange learning experiences that treat each learner as an intrinsically valuable educational end, not as an exploitable means" (p. 20).

Komoski is, however, realistic about the time and training requirements of the reforms he is proposing. He notes that, "neither teachers nor administrators have information or information/management tools equal to the job society is expecting them to do" (p. 24). He continues, "a major implication of these shifts in types and numbers of instructional options available to schools is the problem of sorting out, identifying, correlating, and effectively using the most relevant of these options to fill a particular instructional need in a school's curriculum" (p. 25).

Finally, in the last few pages, not even hinted at before that point, Komoski's purpose becomes clear. He could have labored less in supportinghis arguments and still have convinced the reader to consider the merits of an integrated set of data bases which appear to be extremely beneficial in helping educators to make the reforms he has outlined. These databases, for which Komoski seems to have had a primary developmental responsibility, are entitled the Integrated Instructional Information Resource (IIIR), but are 
referred to simply as the Resource. The Resource provides a comprehensive, evolving set of curriculum descriptors to support a school's work in designing or revising its curriculum purposes, goals and objectives. These descriptors may be used as an aid to:

* building locally developed curricula. By using this adaptable set of descriptors on a special curriculum design spreadsheet, curriculum committees can explore "what ifs" and continually order and reorder a school's curriculum, subject area, grade by grade;

- analyzing, and comparing the subject matter content, and the cognitive processes embedded in textbooks, other learning materials and tests to the content and processes called for in a school's curriculum;

* documenting and tracking the evolution of curriculum thinking and practice over time within a district, a state, or across states;

- using state and nationally recommended curriculum standards to inform local curriculum development; and

- accessing information concerning the need to assess how well a school's curriculum goals are beingmastered by learners (e.g., information about relevant norm-referenced and criterion-referenced tests) providing a structure for accessing, correlating and aligning test information with a school's curriculum goals, its materials, and nonmatierals-based teaching strategies. (pp. 27-28)

The Resource can also aid in:

accessing information on mediated learning experiences ranging from textbooks and the proliferating array of other instructional materials (computer-based, video-based, and print-based), to the increasing numbers of integrated systems, some of which combine computer-aided instruction and management with print and other media; and

accessing information about nonmaterials-based learning experiences and about the ways teachers can organize use of materials to go beyond their obvious uses. This function includes such things as teacher-generated strategies, student studies of nature, local government, their own behavior, as well as having students carry out useful projects with their school and local community (p. 28) 
In addition, creators of the Resource have developed a network of cooperating teaching-traininginstitutions to encourage the use of the Resource among preservice teachers and practicing teachers. There is interest in finding ways in which the Resource may be useful to parents as a way to become more informed about their child's formal education and how they can support efforts at home.

Komoski's criticisms seem unduly harsh at times and his generalizations far too sweeping, but the basic premises which he argues have been reiterated by countless others. Much of what he describes must ring true with those who have spent time in American schools where countless numbers of students spend much of their school day occupied with uninteresting, often trivial busywork; where the effectiveness of the teacher is often evaluated primarily on whether or not students are seated and quiet. Educators from countries other than the United States must judge whether Komoski's comments are appropriate to practices in their schools.

Komoski's views were quite clear long before he chose to free the reader from the continuation of his arguments; a briefer account would have been welcome. Instead, a discussion of the many existing examples of American schools which have worked toward the reforms that Komoski promotes would have been more interesting. Information about schools which have already made use of the Resource would have lent even more credibility to what appears to be a most useful resource.

REVIEWER

Cheryl Amundsen is an Assistant Professor in the Department of Educational Psychology at McGill University, Montreal, Quebec. 


\section{Information for Authors}

CJEC welcomes papers on all aspects of educational communication and technology. Topics include, but are not limited to: media and computer applications In education, leaming resource centers, communication and instructional theory, instructional design, simulation, gaming and other aspects of the use of technology in the leaming process. These may take the form of reviews of literature, descriptions of approaches or procedures, descriptions of new applications, theoretical discussions and reports of research.

\section{Manuscript Categories}

Manuscripts may fall into one of two classes: General, dealing with a topic or issue at a general level (although reference to specific instances or examples may be included), and Proflles, dealing with or describing only a specific instance of an approach, technique, program, project, etc. A Profile may be thought of as a descriptive case study.

Most manuscripts dealing with a topic in general should include reference to supportive literature, while manuscripts submitted to the Profile category may or may not. The Editor reserves the right to change the designation of a manuscriptor to make a designation, if none has been made previously by the author. Authors interested in determining the suitability of materials should consult past issues of CJEC or contact the Editor.

All manuscripts received by the Editor (either general or profile) will be judged for suitability, contribution, accuracy, etc. by a panel of anonymous reviewers designated at the time of submission, Nomally, the revlew process requires about eight weeks. There are no deadlines for the submission of manuscripts.

\section{Manuscript Preparation}

Manuscripts should be typed on $81 / 2 \times 1$ l-inch ordinary white paper. All materials must be doublsspaced, including quotations and references, Include a title page on which appears the title of the manuscript, the full name of the author(s) along with position and institutional affiliation, mailing address and telephone number of the contact author. An abstract of 75-150 words should be placed on a separate sheet following the title page. White the title should appear at the top of the first manuscript page, no reference to the author(s) should appear there or any other place in the manuscript, Elements of style, including headings, tables, figures and references should be prepared according to the Publication Manual of the American Psychological Association, 3rd Edition, 1983. Figures must be camera-ready.

\section{Submission of Manuscripts}

Send fourcopies of the manuscript to the Editor along with a letter stating that the manuscript is original material that has not been published and is not currently being considered for publication elsewhere. If the manuscript contains copyright materials, the author should note this in the cover letter and indicate when letters of pemission will be forwarded to the Editor. Manuscripts and editorial correspondence should be sent to: Robert M. Bemard, Canadian Journal of Educational Communication, Education Department, Concordia University, 1455 de Maisonneuve Blvd. W., Montreal, PQ, H3G 1M8. 